\title{
The B2-B19' orientation relationships and their continuums revealed by EBSD and TKD observations of NiTi alloys
}

A confirmation that we must revise the PTMC

Cyril Cayron

Laboratory of ThermoMechanical Metallurgy (LMTM), PX Group Chair, EPFL

Neuchâtel, Switzerland

cyril.cayron@epfl.ch

$E B S D=$ Electron BackScatter Diffraction

$T K D=$ Transmission Kikuchi Diffraction (= t-EBSD, EFSD)

$O R=$ Orientation Relationship

PTMC = Phenomenological Theory of Martensitic Crystallography 


\section{Motivations of the study:}

1. Three years ago, I started to teach "phase transformations" to Bachelor students. Necessarily, I included a part about shape memory alloys, and one of their most classical representative alloy: $\mathrm{Ni}$-Ti. I was teaching as most of us teach this, by showing schematic pictures as the one on the right hand side:

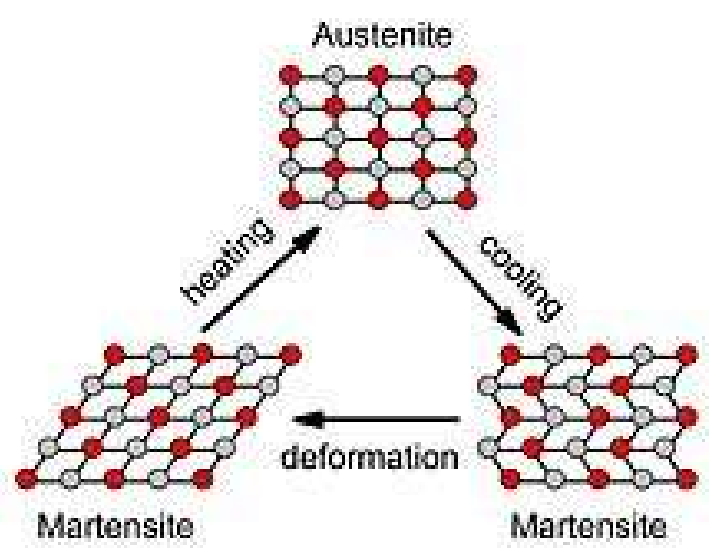

However, as I have a long experience of EBSD, I decided to look inside literature to find experimental EBSD maps showing the B2 austenite $\rightarrow$ B19' martensite transformation during tensile tests of superelastic $\mathrm{NiTi}$, or the $\mathrm{B}^{19} 9^{\prime}$ variant re-orientation effect during deformation of a martensitic NiTi alloy. I knew that NiTi have been widely studied, and I found numerous TEM and HRTEM studies, but to my big surprize I could not find any EBSD map of B19' martensite! So, I decided to do the job myself.

2. The classical theory used to explain/describe the complex microstructure of B19' martensite is the Phenomenolgical Theory of Martensite Crystallography (PTMC) born in the 1950s. I have started to doubt about PTMC and this paradigm nearly 10 years ago when I started to study fcc-bcc martensitic transformation in steels. Before explaining why, let me make a very brief summary of what is PTMC. 
Phenomenological Theory of Martensite Crystallography (1/3)

Version 1: Bowles \& Mackenzie 1954

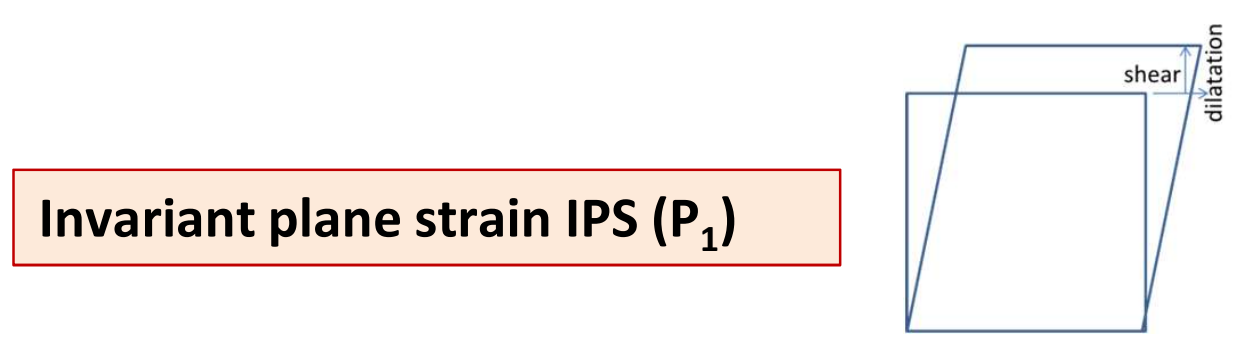

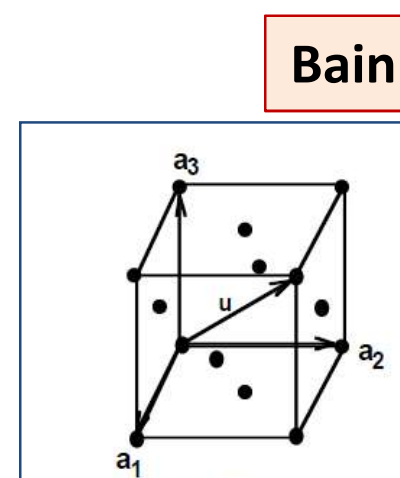

(a)

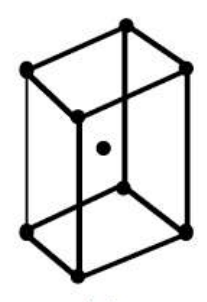

(c)
Body-centered

tetragonal austenite

From HKDH Bhadeshia

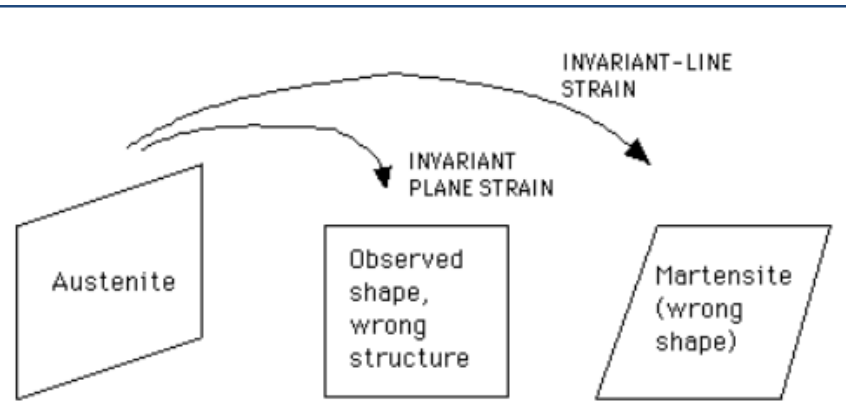

(b)

(c)

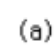

(a) - (b)

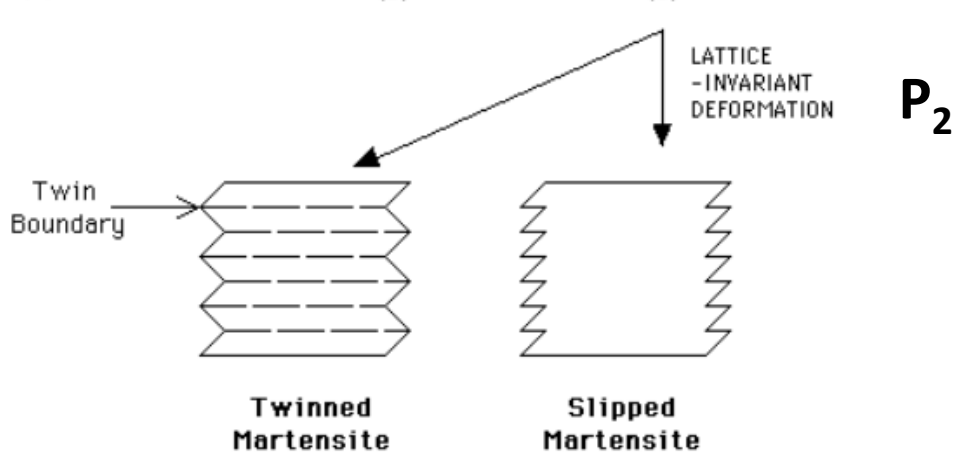

Correct macroscopic shape, correct structure

$$
R B=P_{1} P_{2}
$$


Phenomenological Theory of Martensite Crystallography (2/3)

\section{Version 2: Wechsler, Lieberman \& Read 1953}

\section{Mathematically equivalent to version 1}

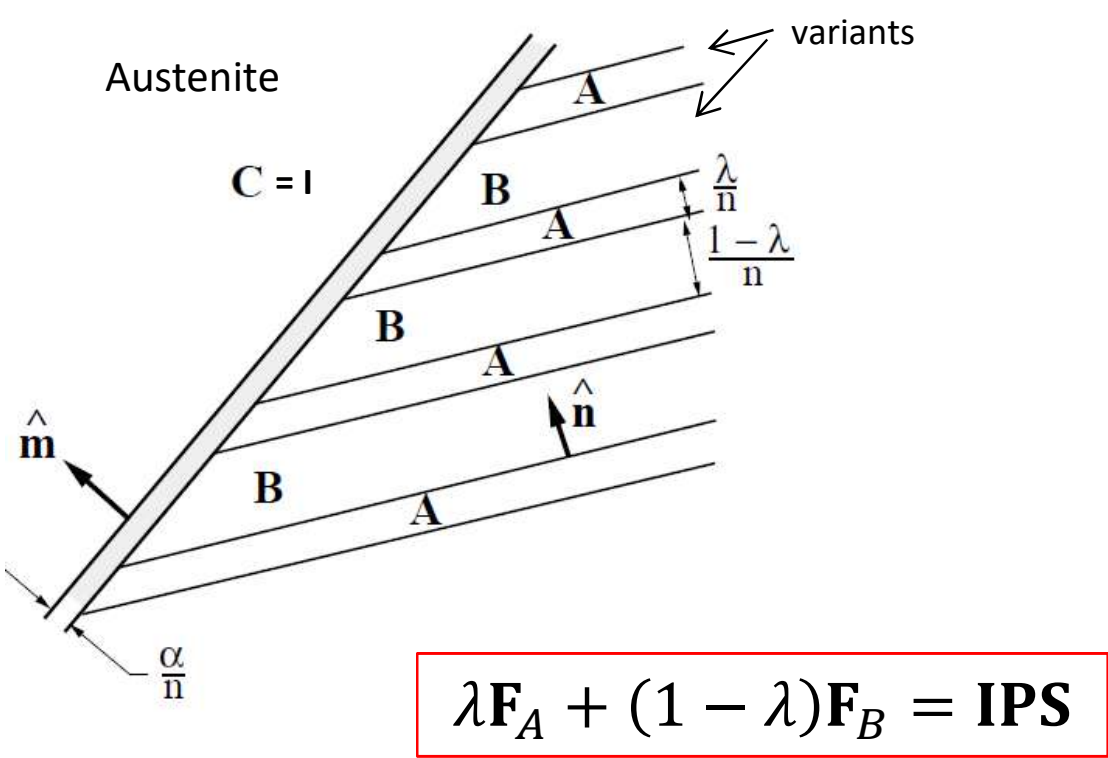

with $\mathbf{F}_{A}$ and $\mathbf{F}_{B}$ two distortion variants

In addition the volume change should be conserved: $\operatorname{det}\left(\mathbf{F}_{A}\right)=\operatorname{det}\left(\mathbf{F}_{B}\right)=\operatorname{det}($ IPS $)$
“Modern" form: Ball \& James, Bhattacharya

Compatibility condition:

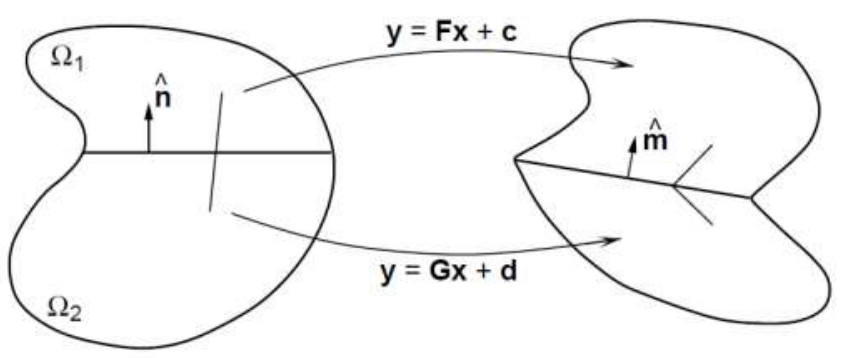

The interface plane $(\mathrm{n})$ is distorted similarly by $\mathrm{F}$ and $\mathrm{G}$

$$
\mathbf{F}-\mathbf{G}=\boldsymbol{a} \otimes \boldsymbol{n}
$$

$\mathrm{F}$ and $\mathrm{G}$ are rank-one connected

$$
\Leftrightarrow \quad \mathbf{F}_{A}-\mathbf{F}_{B}=\boldsymbol{a} \otimes \boldsymbol{n}
$$


PTMC suffers from important problems:

- They are phenomenological (they do not describe how the atoms move)

- They rely on the exact values of the lattice parameters, which, to me, has never been not confirmed by experience.

- The equations lead to some solutions that are discarded just because they do not fit with the experiments reported in literature.

- They are not as "predictive" as it is often claimed.

- The rotational degree(s) of freedom noted $R$ or $Q$ depends on the pair of "correspondence" variants (actually stretch variants) that is considered! In the modern version of PTMC, they are

$$
\left\{\begin{array}{l}
\text { Rotation } \mathbf{Q}_{i j} \text { required to get a coupling between different variants } i \text { and } j \\
\text { Rotation } \mathbf{J}_{i j k l} \text { required to get a coupling the habit plane variants }(i, j) \text { and }(k, l) \\
\rightarrow \text { Very complex. When does it stop? }
\end{array}\right.
$$

- They are intrinsically built on the hypothesis that the twinning relation between the variants should be a simple shear. 


\section{Shifting the simple shear paradigm (1/5)}

The main issue for simple shear: the atoms in the lattice ! Friction due to steric effect, one should expect $\tau_{c} \# \mu / 30$ if lattice distortion is an "instantaneous" simple strain

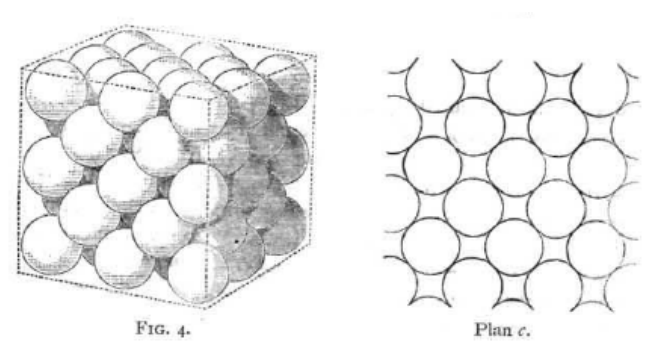

Barlow, Nature, 1883 (a)

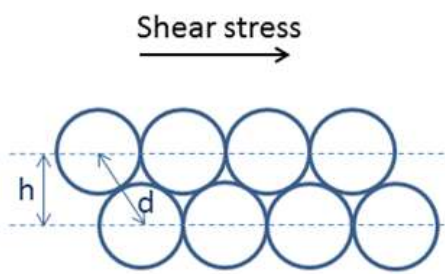

Parent crystal
Twinning by simple shear

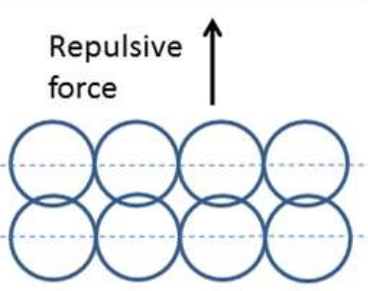

Intermediate state

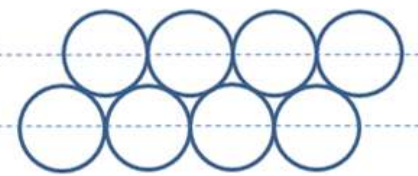

Twinned crystal

\section{Hypothesis 1: dislocations (1940-now)}

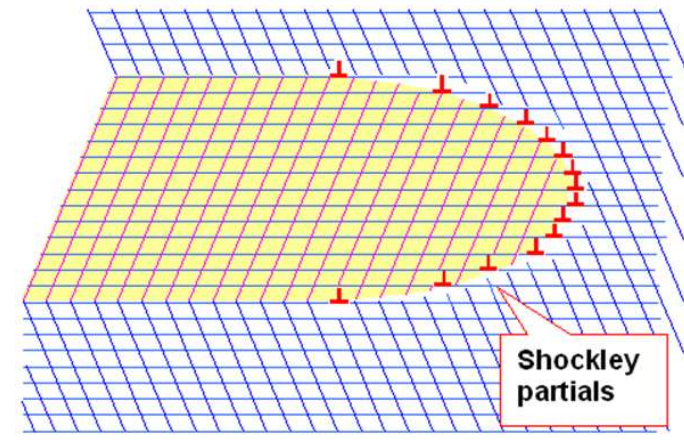

$\underline{\text { origin }}=$ hypothetical pole mechanism

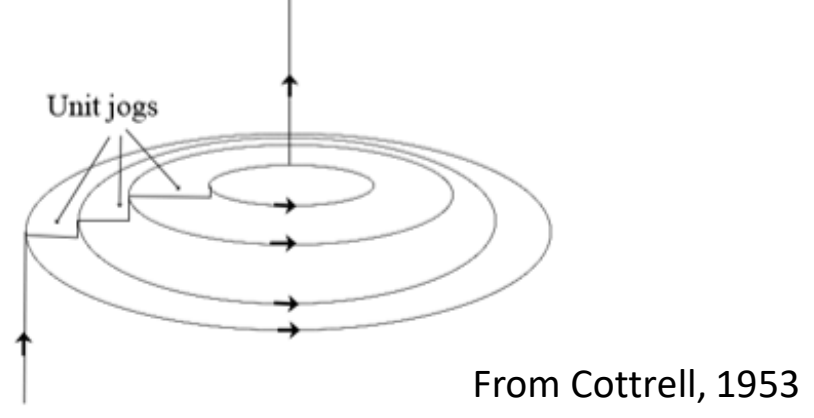

Hypothesis 2: simple shear should be replaced by a more general concept: angular distortion introduced for fcc-bcc transformation (2015), and generalized to fcc-hcp-bcc transformations and deformation twinning.

(b)

\section{Twinning by angular distortion}

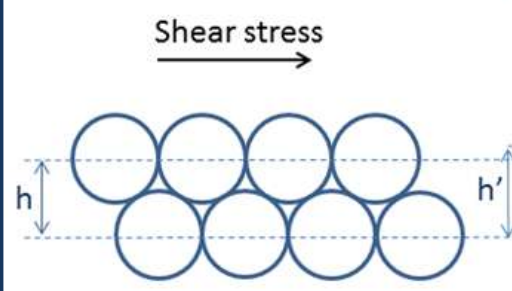

Parent crystal

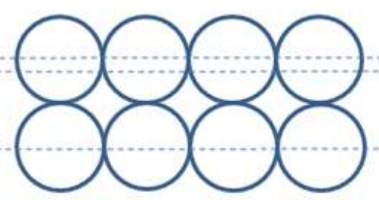

Intermediate state

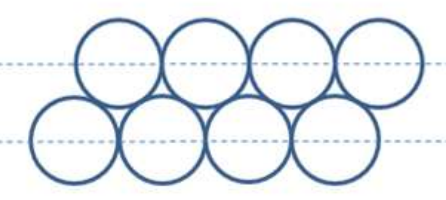

Twinned crystal

C. Cayron, Crystals 2018, 8, 181; doi:10.3390/cryst8040181 


\section{Hypothesis 2:}

The initial problem comes from the current assumption that all the atoms should move «instantaneously», which is impossible

\section{Phase transformation / deformation imagined as a wave (soliton)}

\section{time}
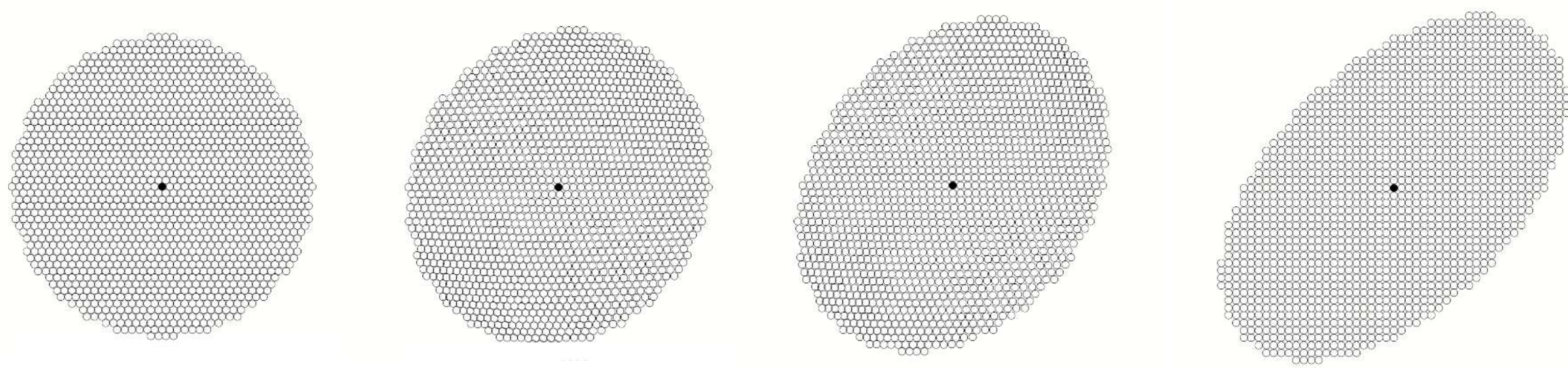

- The lattice distortion is not based on simple strain, or IPS, as in PTMC.

- No dislocation, no disconnection involved in the mechanism.

- This "transformation wave" can go at the speed of sound.

- Accommodation is spread on large distances. It can be discretised into sets of dislocations/disconnections at lower speeds or in small volumes (when the martensite product or the twin reaches an interface). 


\section{Hexagonal phase} surrounding matrix

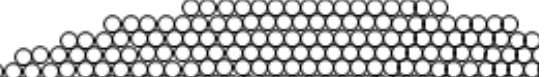

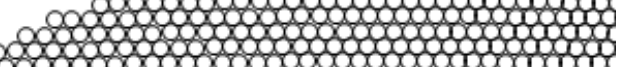

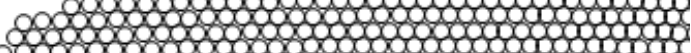

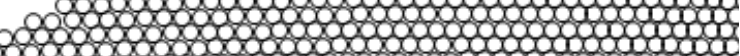

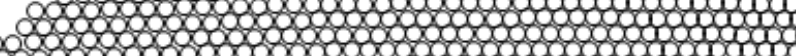
10000000000000 \$2

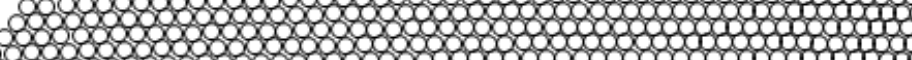
O L 8

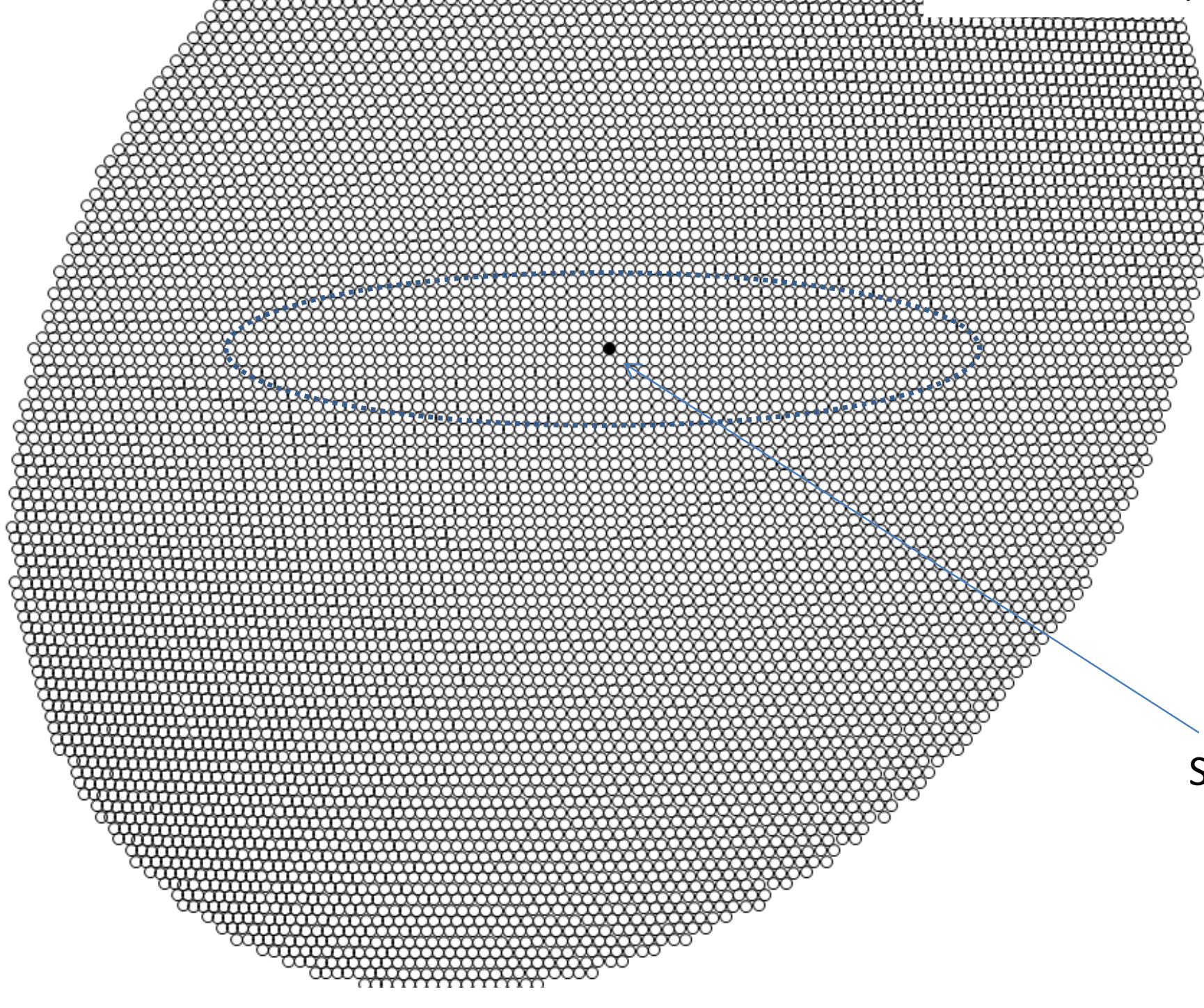

Accommodation zone: Set of infinitesimal disclinations + disconnections?

Becomes a set of disconnections at low speed or when the martensite/twin stops?
Square phase core 
Shifting the simple shear paradigm (4/5)

\section{Shift of paradigm: angular distortion instead of simple strain}

My point of view: Dislocations are just accommodation defects generated by the formation of martensite in the surrounding austenite; they are not intrinsically essential to the transformation. In small free crystals, the transformation can be imagined without dislocation.
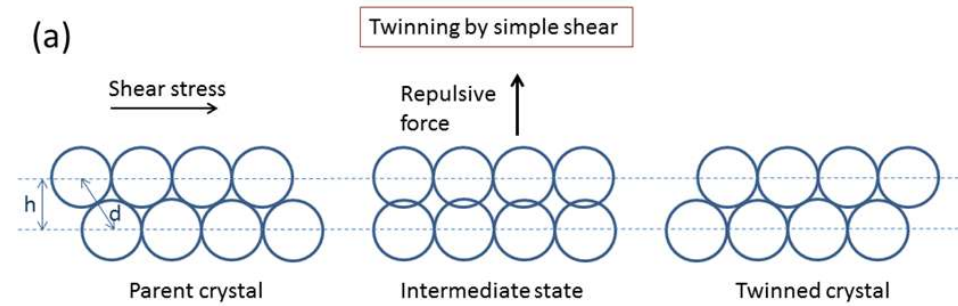

(b) Twinning by angular distortion
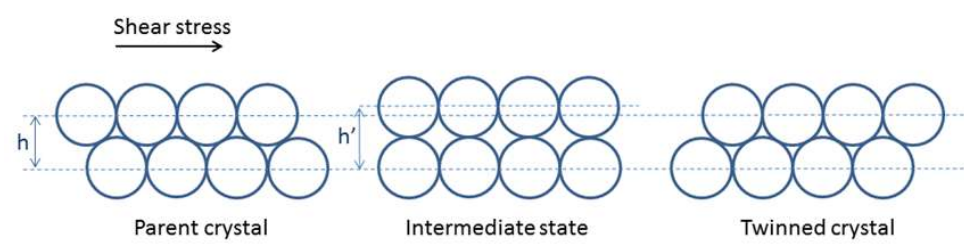

(a)

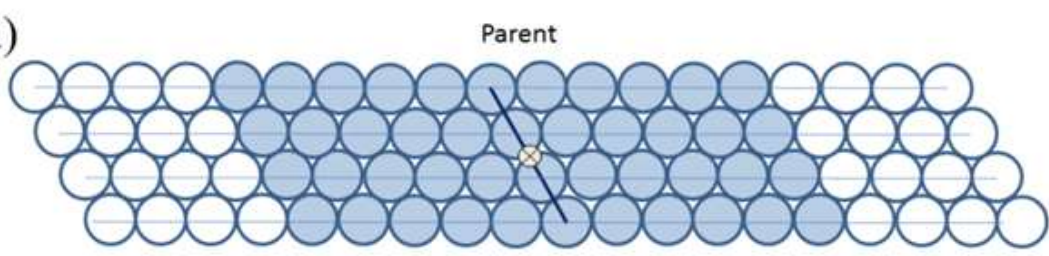

(b)

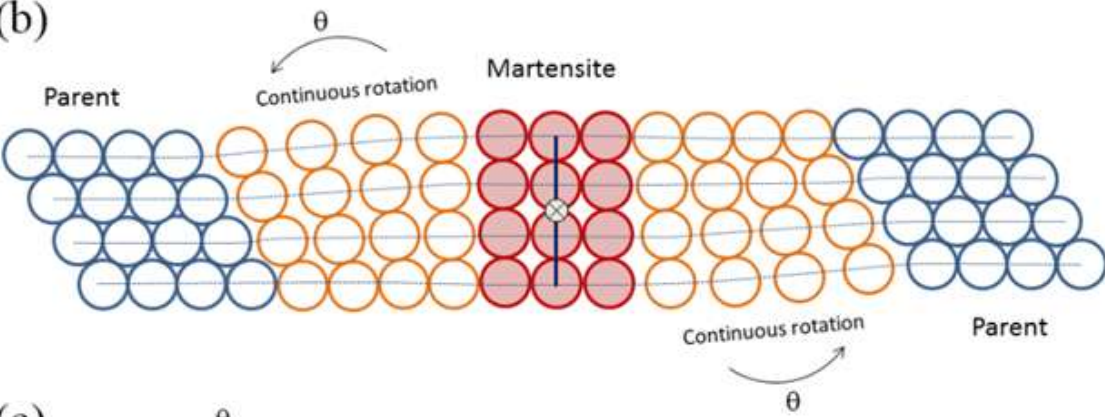

(c)

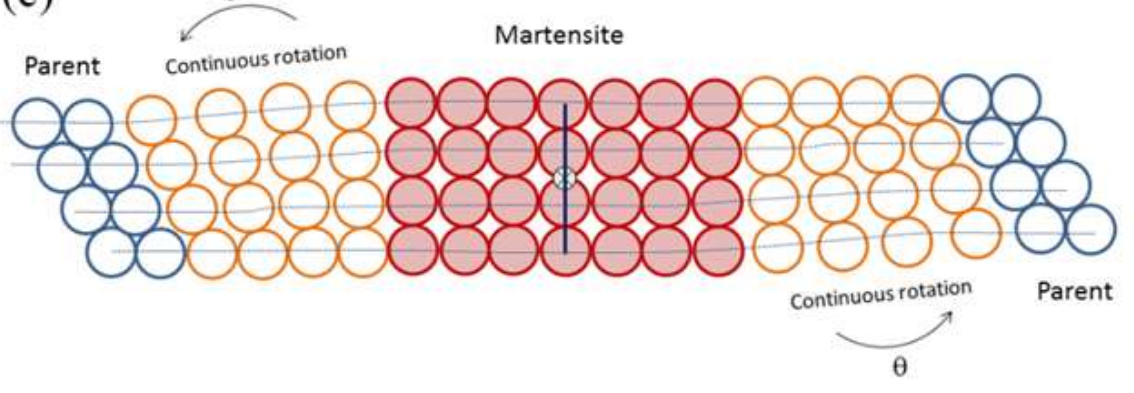

Cayron, C. Shifting the Shear Paradigm in the Crystallographic Models of Displacive Transformations in Metals and Alloys. Crystals. 2018, 8, 181. 


\section{Shifting the simple shear paradigm (5/5)}

Application to fcc-bcc martensitic transformation in steels
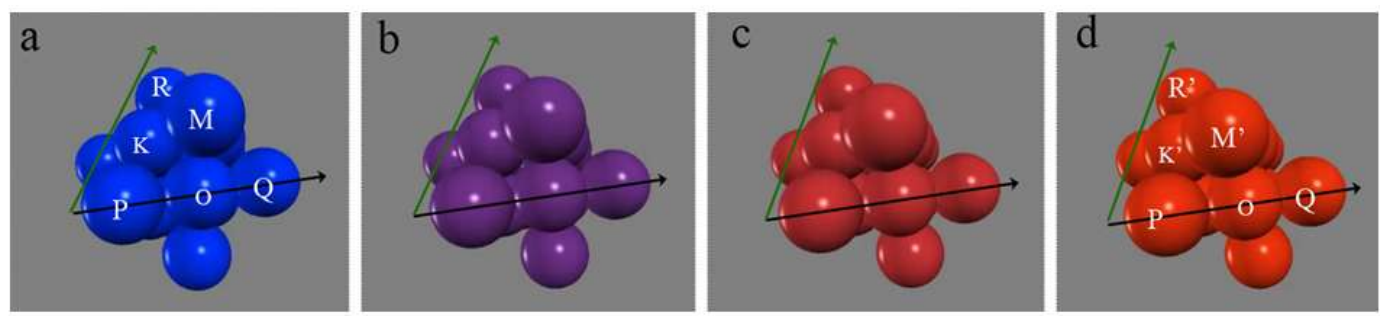

Fcc-bcc distortion with K-S OR explained with hard-spheres. The continuity is given by a unique parameter, the angle, $\beta=(\mathbf{P O}, \mathbf{P K})$ that changes from $60^{\circ}$ (bcc),$X=1 / 2$, to $70.5^{\circ}$ (fcc), $X=1 / 3$. Movie at http://Imtm.epfl.ch/research.

Application to conventional and unconventional deformation twinning in magnesium

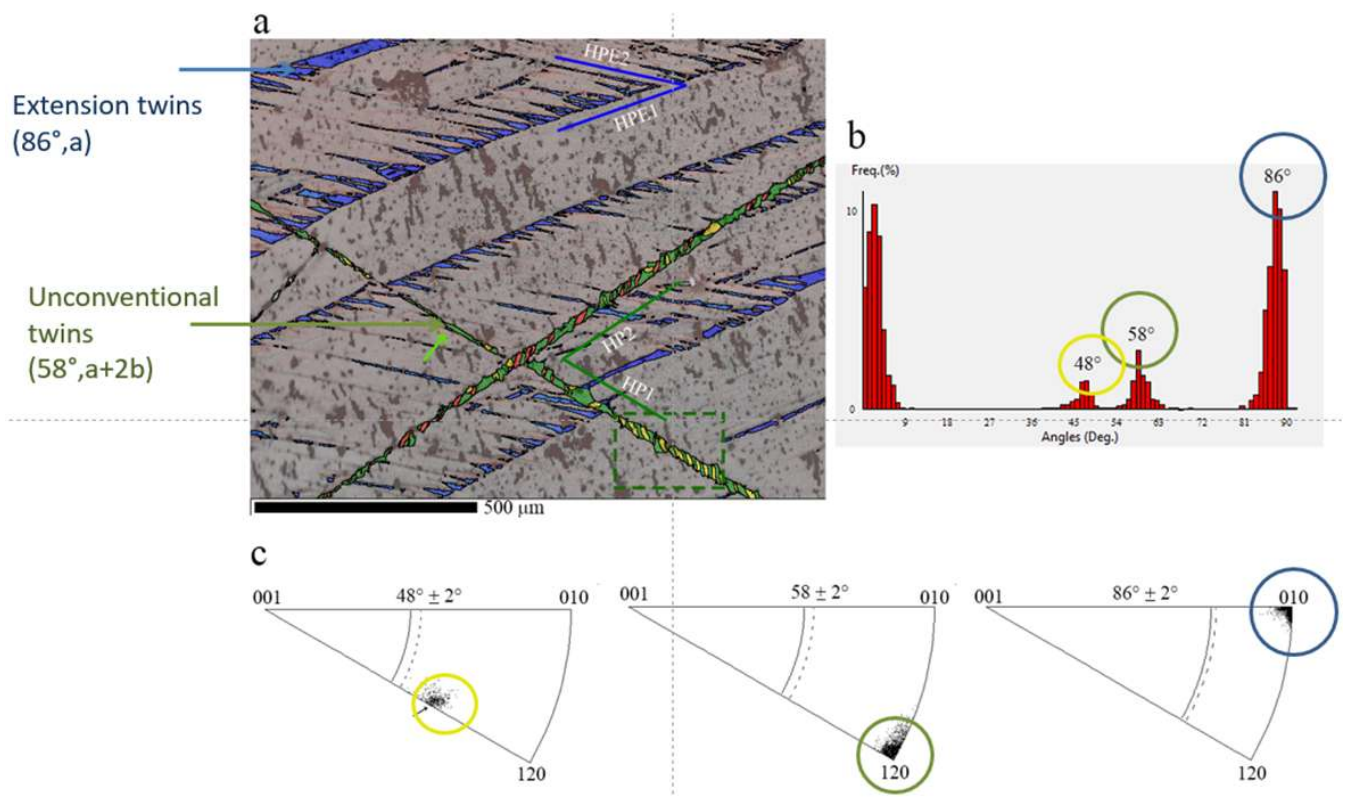

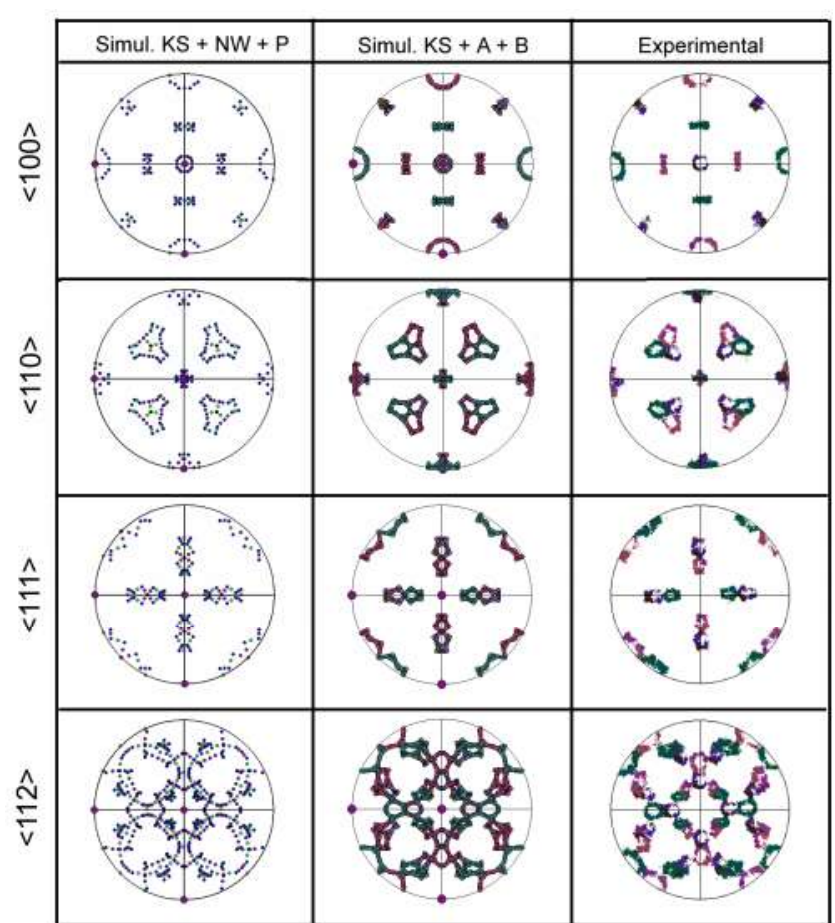

The habit plane of the green twin is $\{10 \overline{1} 2\}_{\mathrm{t}} / /\{21 \overline{3} 2\}_{\mathrm{p}}$ $\rightarrow$ It is not a invariant plane, so it is not a shear plane!

Cayron, C. Shifting the Shear Paradigm in the Crystallographic Models of Displacive Transformations in Metals and Alloys. Crystals. 2018, 8, 181. 


\section{Methodology for the EBSD and TKD on NiTi alloys}

Material:

- Straight 50Ni-50Ti bars of $10 \mathrm{~mm} \times 2 \mathrm{~mm}$ sections were used in this study.

- $\mathrm{Ms}=45^{\circ} \mathrm{C}, \mathrm{Mf}=35^{\circ} \mathrm{C}, \mathrm{As}=60^{\circ} \mathrm{C}$, and $\mathrm{Af}=90^{\circ} \mathrm{C}$.

- The shape memory effect was confirmed by deforming the bars and checking that they come back to their initial straight shapes when dropped into hot water.

\section{Sample preparation:}

- EBSD samples prepared by electropolishing with $\mathrm{A} 3$ electrolyte from Struers at $20 \mathrm{~V}$ and $10{ }^{\circ} \mathrm{C}$, or using a lab-made solution of $10 \%$ perchloric acid $+30 \%$ butanol $+60 \%$ methanol at $30 \mathrm{~V}$.

- TEM samples used for TKD prepared by mechanically polishing down to $100 \mu \mathrm{m}$ and punching to obtain discs of $3 \mathrm{~mm}$ diameter. Then electropolished at $-15^{\circ} \mathrm{C}$ with a dual-jet TenuPol electropolisher from Struers with the $\mathrm{A} 3$ electrolyte at $20 \mathrm{~V}$, or with nitric $30 \%$ acid $+70 \%$ methanol solution at $40 \mathrm{~V}$.

\section{Facilities and conditions:}

- SEM: Gemini450 SEM (Zeiss, Germany).

- EBSD and TKD : CMOS Symmetry system processed with Aztec software (Oxford Instruments, UK).

- Current $15 \mathrm{nA}$ and accelerating voltage $20 \mathrm{kV}$ for EBSD and $30 \mathrm{kV}$ for TKD.

\section{Software for Data treatment:}

- Simulations of the orientation variants and their pole figures: GenOVa

- B2 parent austenite reconstruction, disorientation histogram (angles and axes), traces of the habit planes: ARPGE.

\section{Phases:}

- B2: cubic m3m, a = 3.015 А.

- B19': monoclinic $2 / m, a=2.89 \AA, b=4.12 \AA, c=4.62 \AA$, and $\beta=96.8^{\circ}$ for $B 19^{\prime}$. 


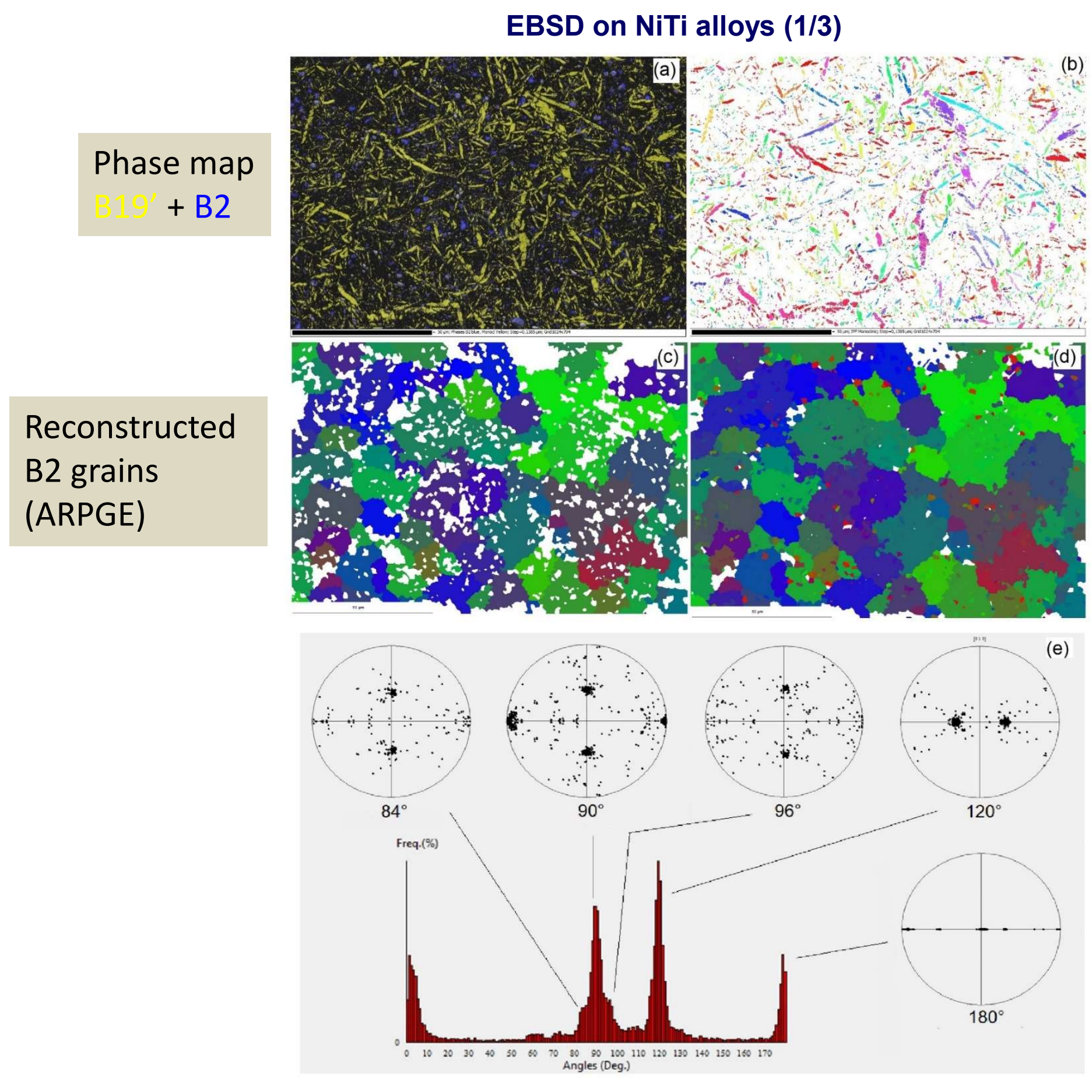

Orientation map of B19'

Reconstructed

B2 grains + retained cubic phases (ARPGE)

Disorientation histogram between the B19' martensite inside their prior parent B2 grains (ARPGE) 


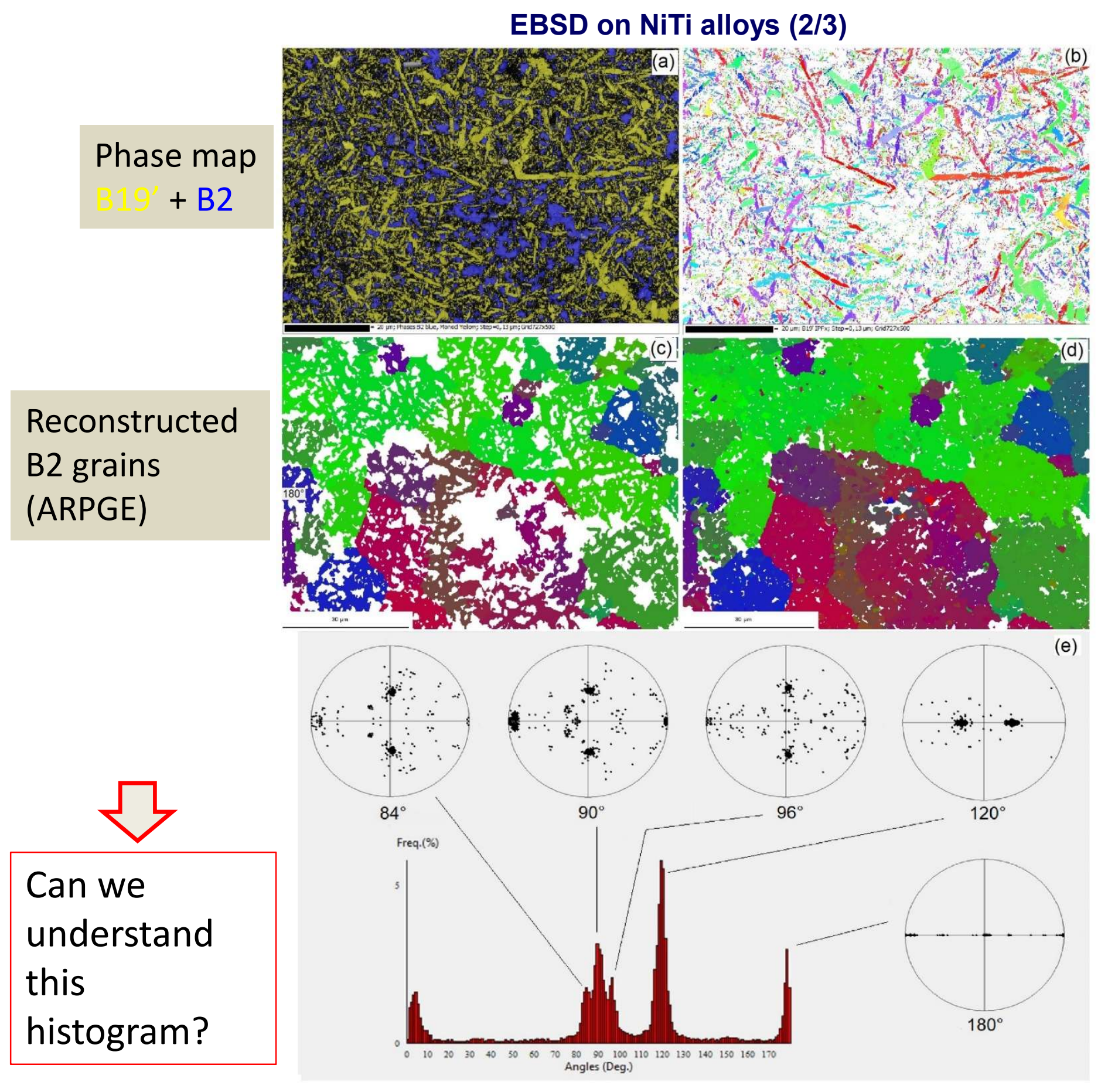

Orientation map of B19'

Reconstructed

B2 grains + retained $B 2$ phases (ARPGE)

Disorientation histogram between the B19' martensite inside their prior parent B2 grains (ARPGE) 


\section{EBSD on NiTi alloys (3/3)}

The B2-B19' orientation relationships (OR) can be determined from the pole figures
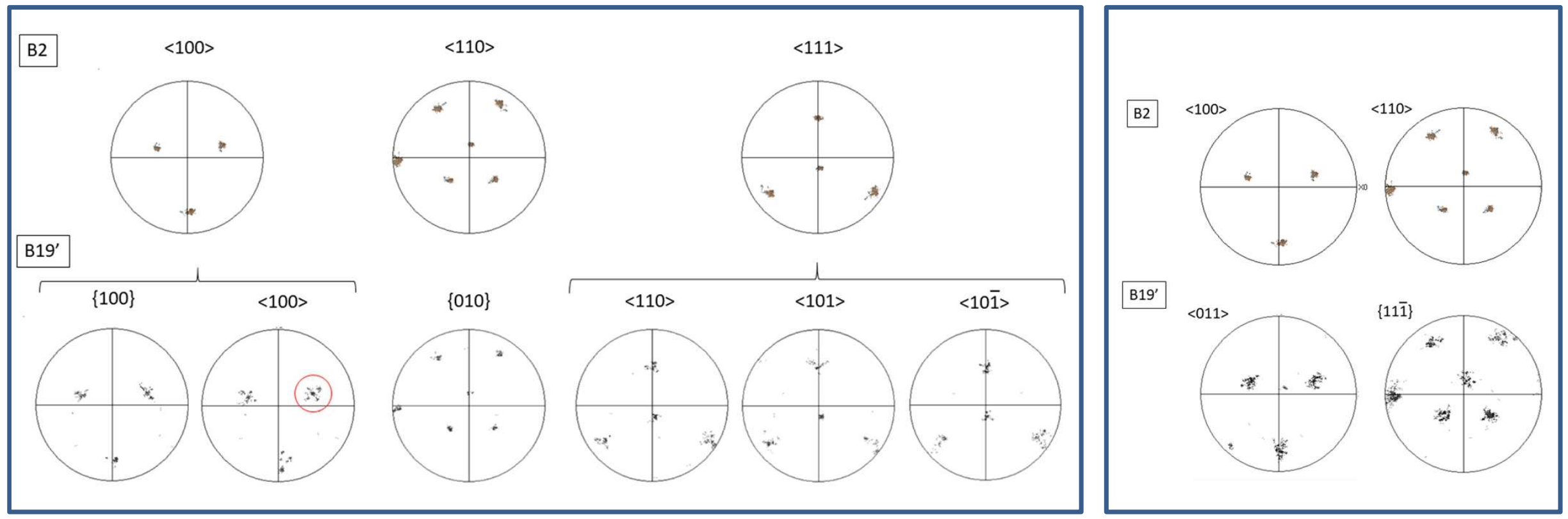

- OR A:

$(010)_{\mathrm{B} 19^{\prime}} / /(110)_{\mathrm{B} 2} \&[001]_{\mathrm{B} 19^{\prime}} / /[110]_{\mathrm{B} 2}$

- OR C:

$(010)_{\mathrm{B} 19^{\prime}} / /(110)_{\mathrm{B} 2} \&[100]_{\mathrm{B} 19^{\prime}} / /[001]_{\mathrm{B} 2}$

- OR AQ:

$(010)_{\mathrm{B} 19^{\prime}} / /(110)_{\mathrm{B} 2} \&[101]_{\mathrm{B} 19^{\prime}} / /[\overline{1} 11]_{\mathrm{B} 2}$

- OR CQ:

$(010)_{\mathrm{B} 19^{\prime}} / /(110)_{\mathrm{B} 2} \&[10 \overline{1}]_{\mathrm{B} 19^{\prime}} / /[\overline{1} 1 \overline{1}]_{\mathrm{B} 2}$

- OR I:

$(11 \overline{1})_{\mathrm{B} 19^{\prime}} / /(101)_{\mathrm{B} 2}$ and $[011]_{\mathrm{B} 19^{\prime}} / /[010]_{\mathrm{B} 2}$ 


\section{Simulation of EBSD pole figures and disorientation histogram (1/3)}

Simulation of the variants associated with the different ORs (GenOVa)

Pole figures of the variants
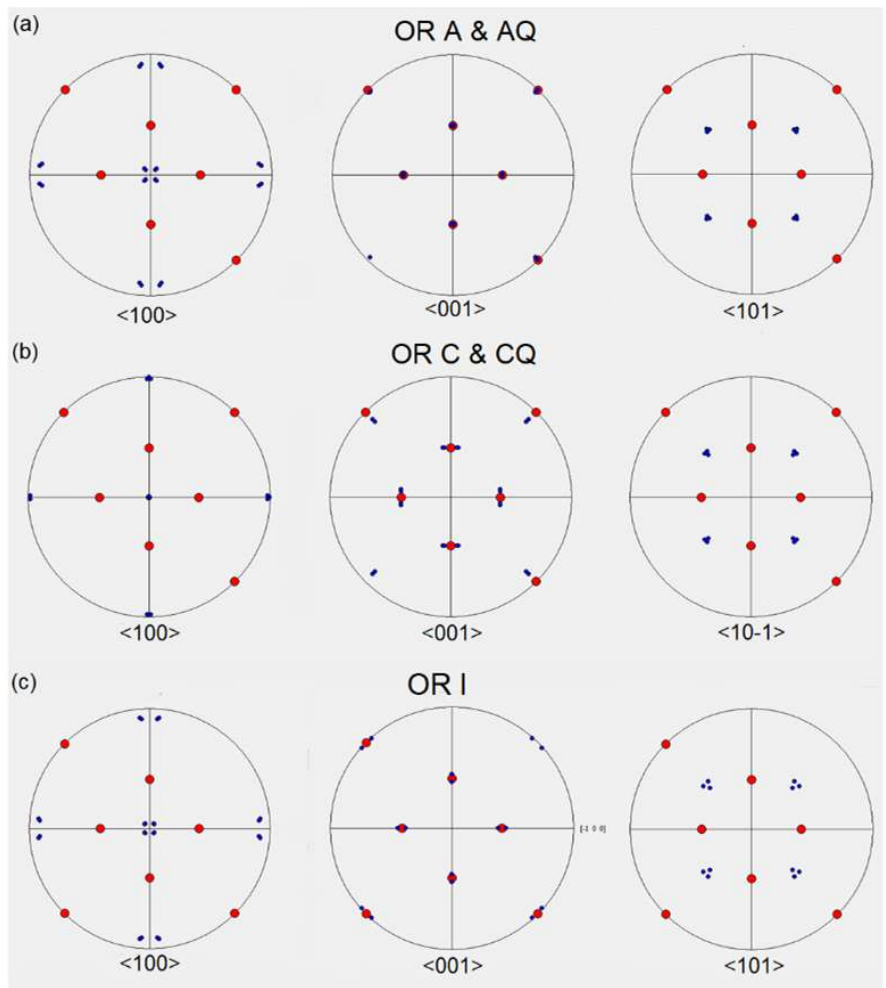

List of operators between variants
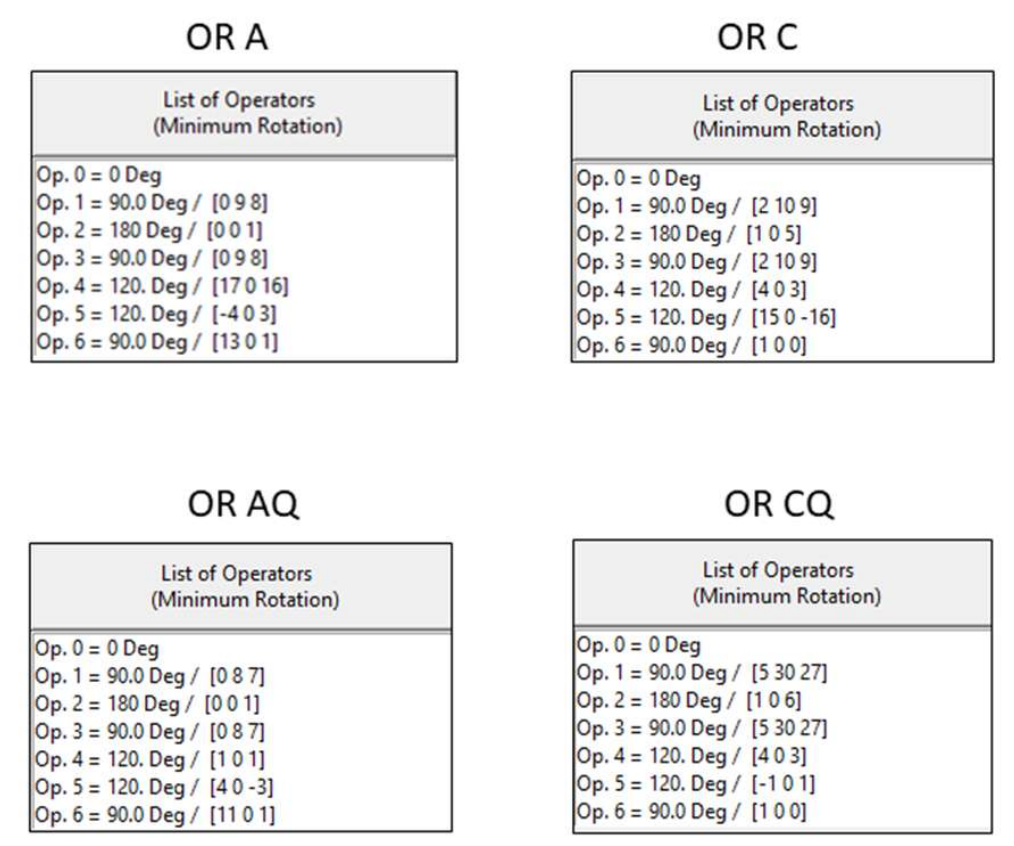

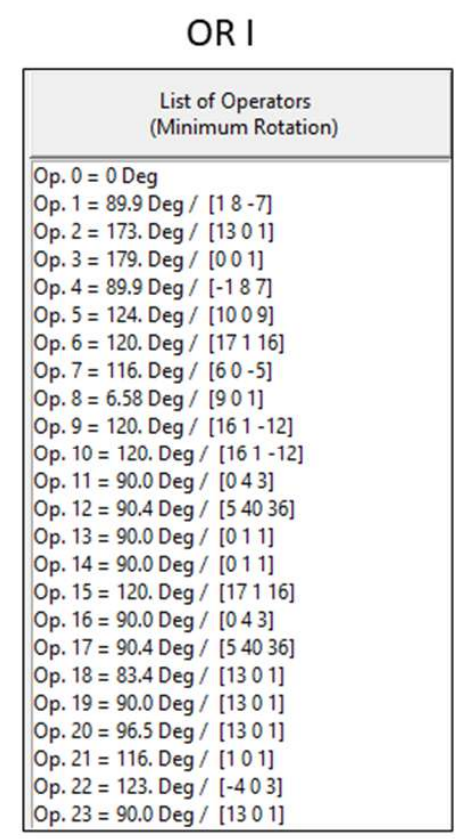

OR A

OR continuum

OR C
Addition of a continuum of Ors between ORA and ORC
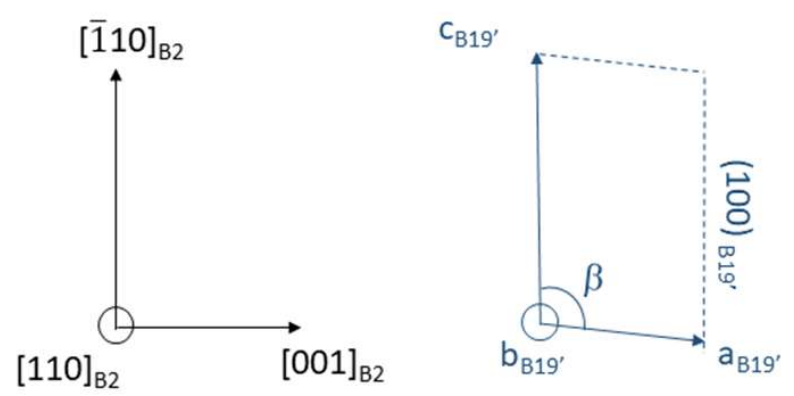

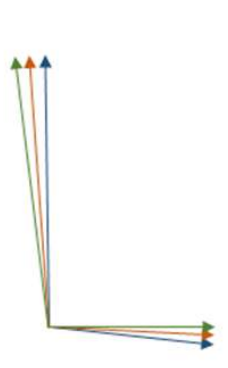

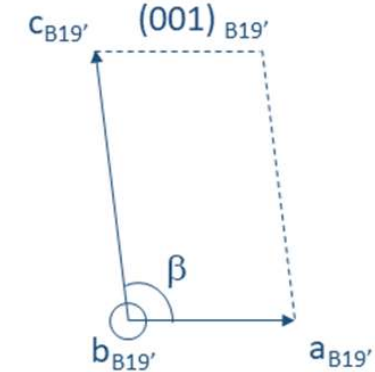


Simulation of EBSD pole figures and disorientation histogram (2/3)

Experimental disorientation histogram

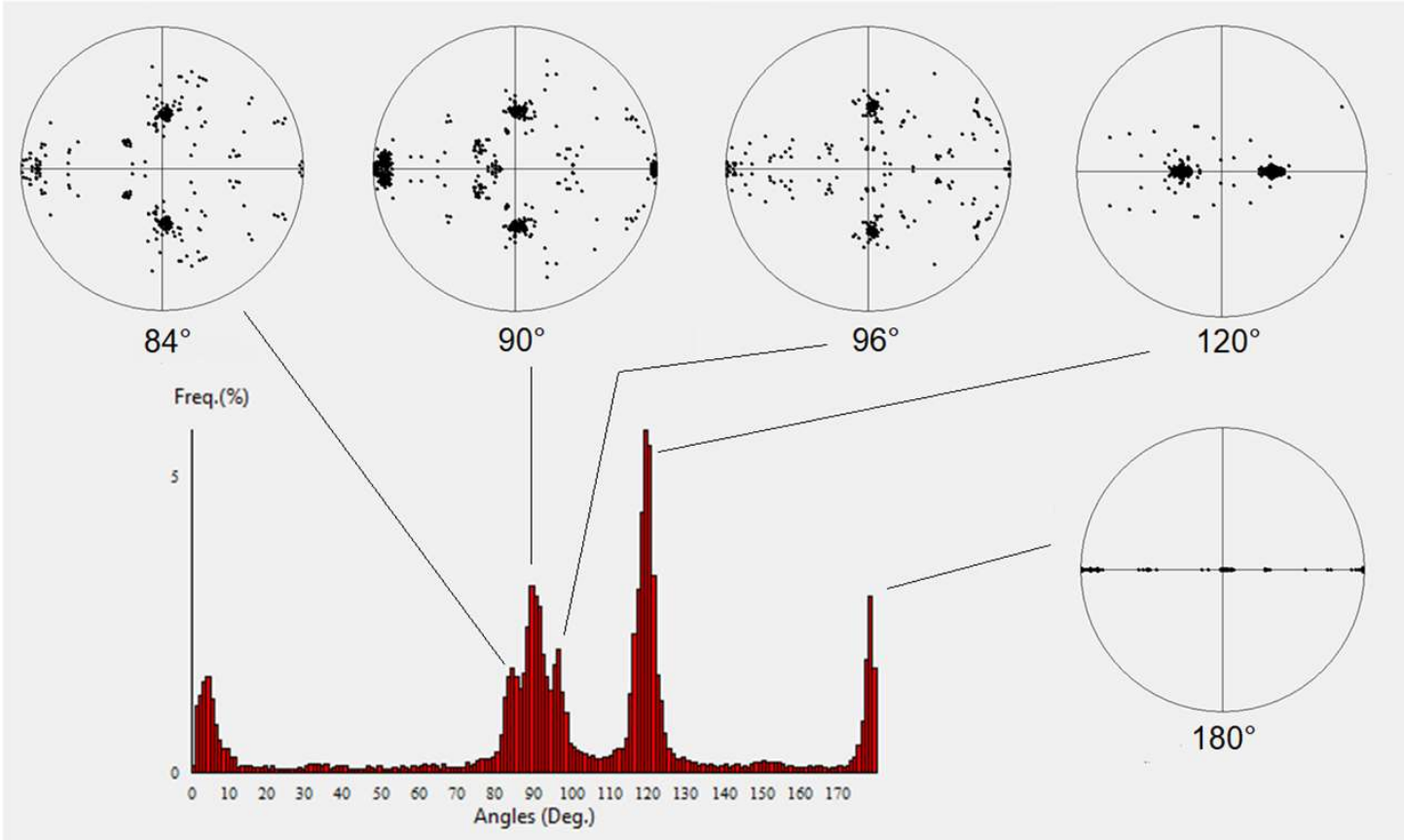

Simulated disorientation histogram with ORs $A, A Q, C, C Q$, and $\mathrm{A}-\mathrm{C}$ continuum

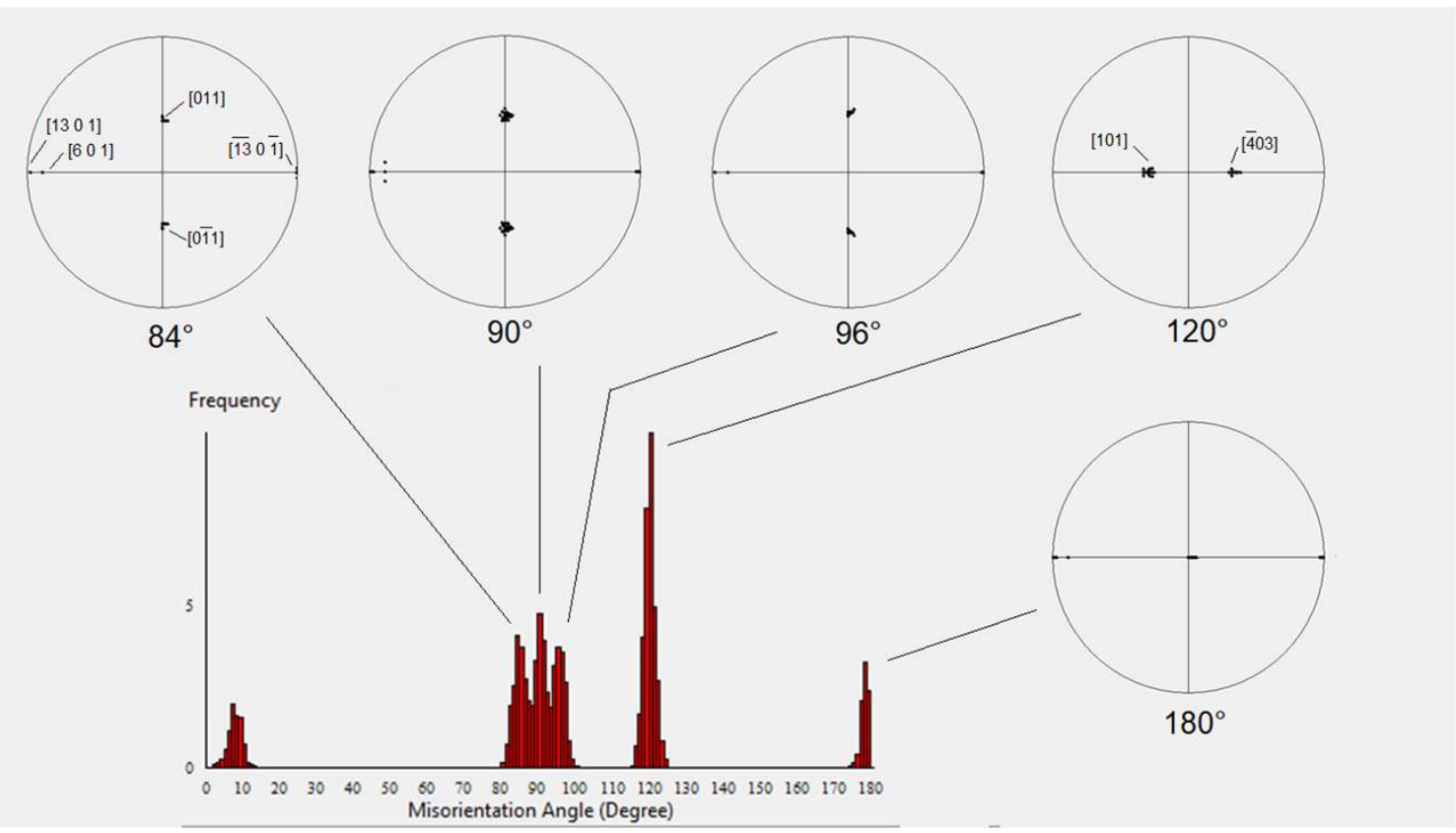


Simulation of EBSD pole figures and disorientation histogram (3/3)

OR A/AQ is the predominant OR in the EBSD map.

ORA is actually that used to illustrate the B2 $\rightarrow$ B19' lattice distortion in Otsuka, K.; Ren, X. Physical metallurgy of Ti-Ni-based shape memory alloys. Prog. Mat. Sci. 2005, 50, 511-678.

OR AQ is for me the "natural" OR because it established a parallelism between the dense planes and dense directions:

$(010)_{\mathrm{B} 19^{\prime}} / /(110)_{\mathrm{B} 2} \&[101]_{\mathrm{B} 19^{\prime}} / /[\overline{1} 11]_{\mathrm{B} 2}$

as the KS OR in bcc martensite in steels, or Burgers OR in hcp martensite in Ti alloys.

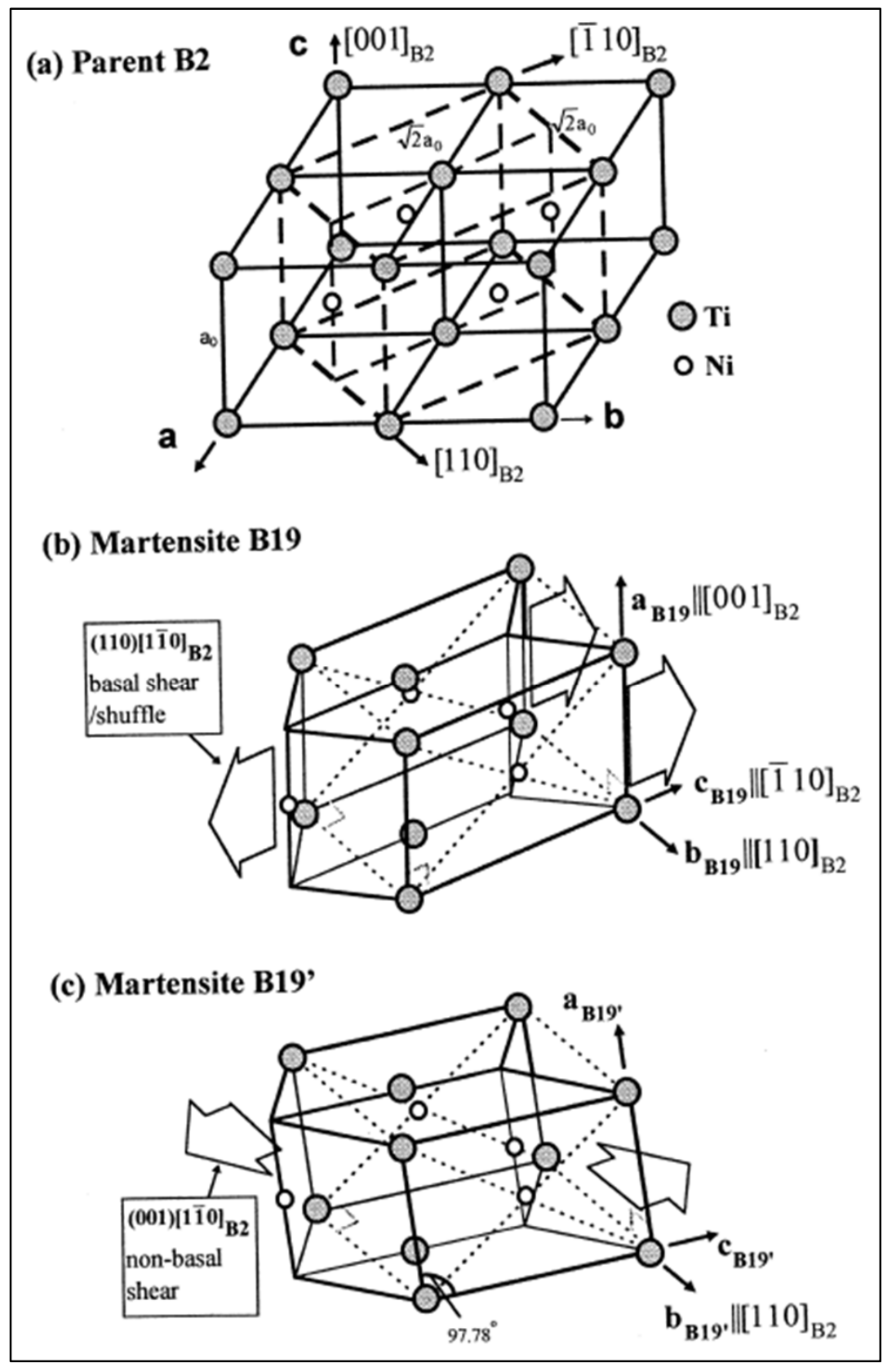




\section{TKD on NiTi alloys (1/3)}

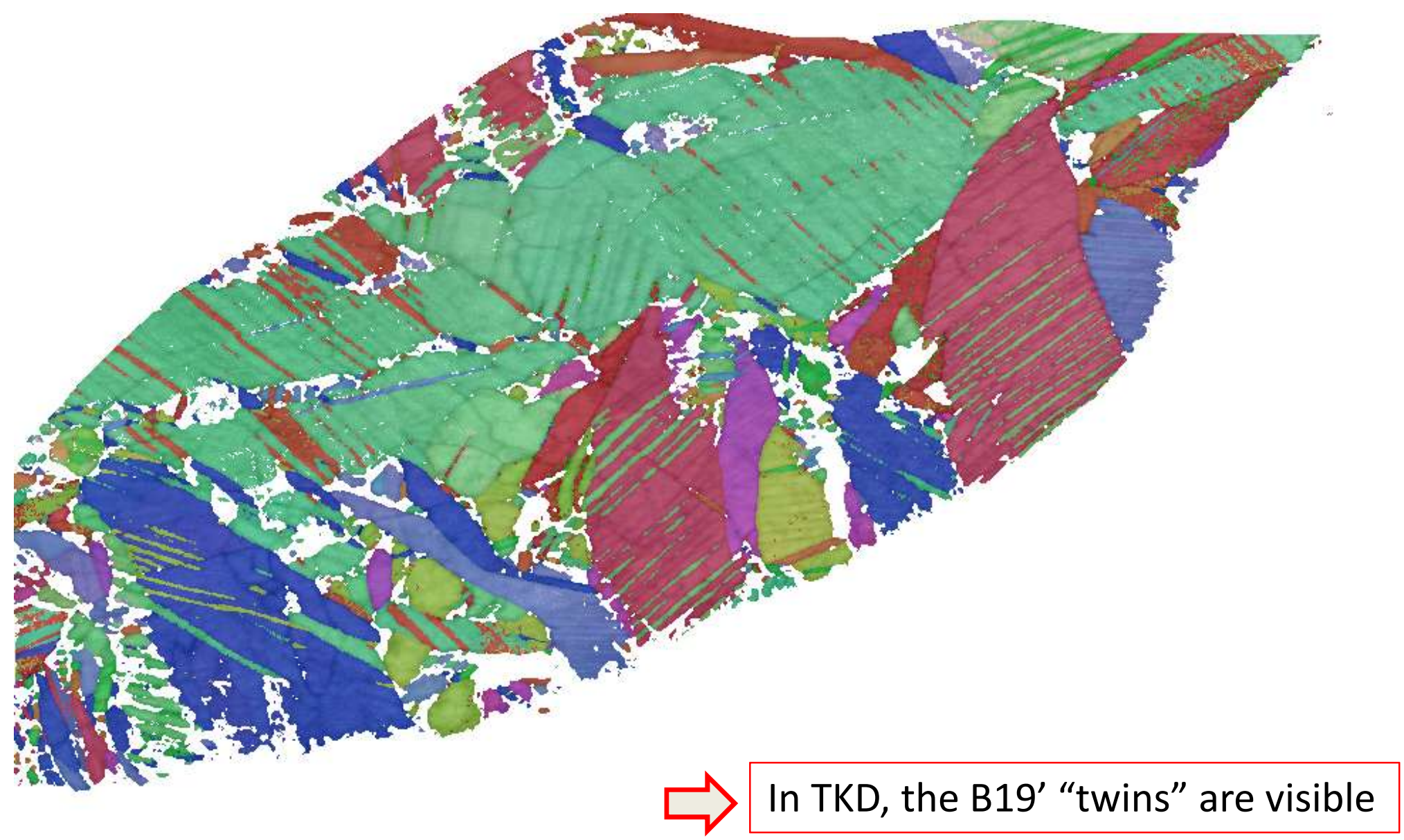


TKD on NiTi alloys (2/3)

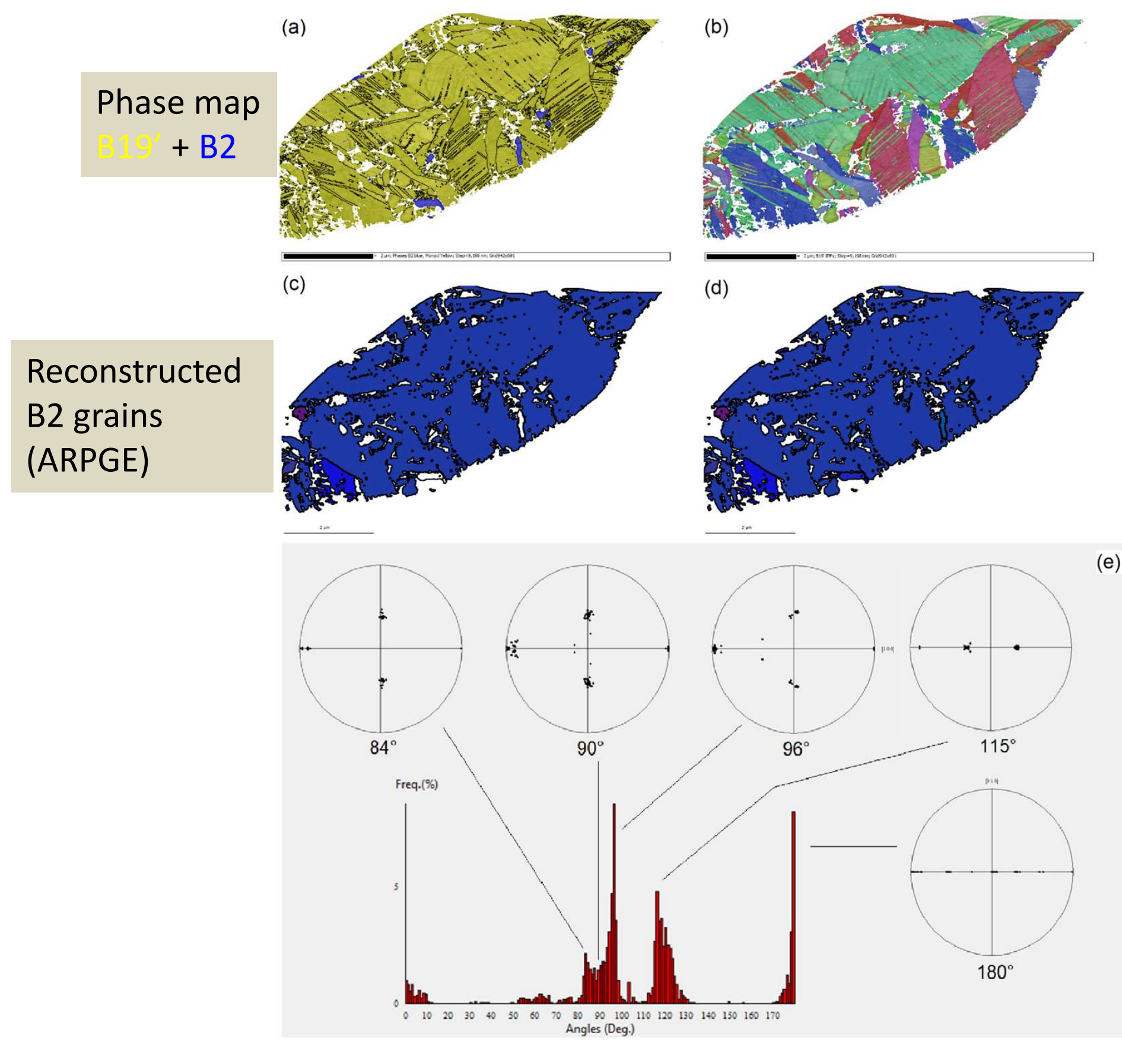

Orientation map of B19'

Reconstructed

B2 grains + retained cubic phases (ARPGE)

(e)

Disorientation histogram between the B19' martensite inside their prior parent B2 grains (ARPGE) 


\section{TKD on NiTi alloys (3/3)}

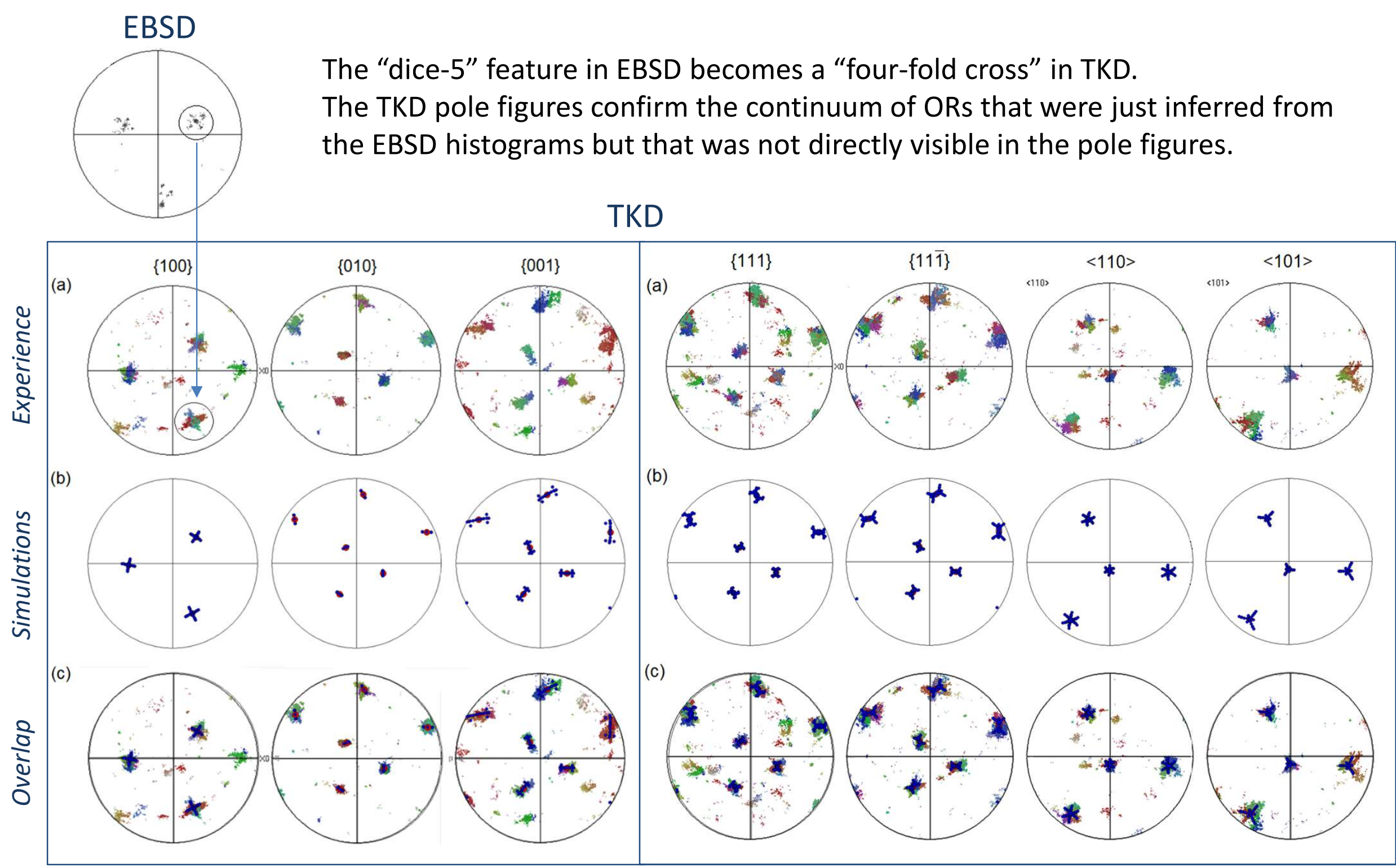

Simulations with all the variants generated by the ORs $A, A Q, C, C Q, I$, and $A-C$ with GenOVa 


\section{Crystallography of B2 $\rightarrow B 19$ ' transformation - without PTMC - (1/4)}

Let us calculate the distortion matrices associated to the different ORs, and let us see if we can predict the habit planes (with big hopes for ORA).

1. The correspondence matrix is the same for all the ORs $A$, $A Q, C, C Q, I$ (because they are all very close). It is:

$$
\mathbf{C}^{\mathrm{B} 2 \rightarrow \mathrm{B} 19 \prime}=\left[\begin{array}{ccc}
0 & 1 & -1 \\
0 & 1 & 1 \\
1 & 0 & 0
\end{array}\right] .
$$

2. The orientation relationship matrix depends on the OR. Let us calculate it for ORA, by considering again Otsuka and Ren scheme.

$$
\mathbf{T}_{A}^{\mathrm{B} 2 \rightarrow \mathrm{B} 19 \prime}=\left[\mathcal{B}^{\mathrm{B} 2} \rightarrow \mathcal{B}^{\mathrm{B} 19}\right]\left[\mathcal{B}^{\mathrm{B} 19} \rightarrow \mathcal{B}^{\mathrm{B} 19 \prime}\right]
$$

$$
\begin{aligned}
& {\left[\mathcal{B}^{\mathrm{B} 2} \rightarrow \mathcal{B}^{\mathrm{B} 19}\right]=\left[\begin{array}{ccc}
0 & \frac{1}{\sqrt{2}} \frac{b_{B 19 \prime}}{\mathrm{a}_{B 2}} & \frac{-1}{\sqrt{2}} \frac{c_{B 19 \prime}}{\mathrm{a}_{B 2}} \\
0 & \frac{1}{\sqrt{2}} \frac{b_{B 19 \prime}}{\mathrm{a}_{B 2}} & \frac{1}{\sqrt{2}} \frac{c_{B 19 \prime}}{\mathrm{a}_{B 2}} \\
\frac{a_{B 19 \prime}}{\mathrm{a}_{B 2}} & 0 & 0
\end{array}\right]} \\
& {\left[\mathcal{B}^{\mathrm{B} 19} \rightarrow \mathcal{B}^{\mathrm{B} 19 \prime}\right]=\left[\begin{array}{ccc}
\operatorname{Sin}\left(\beta_{B 19 \prime}\right) & 0 & 0 \\
0 & 1 & 0 \\
\operatorname{Cos}\left(\beta_{B 19 \prime}\right) & 0 & 1
\end{array}\right] \text {. }}
\end{aligned}
$$

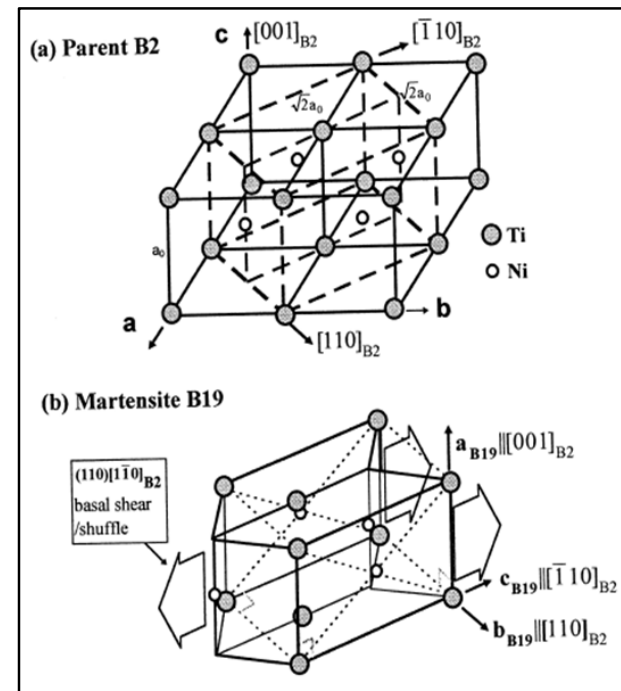

(c) Martensite B19

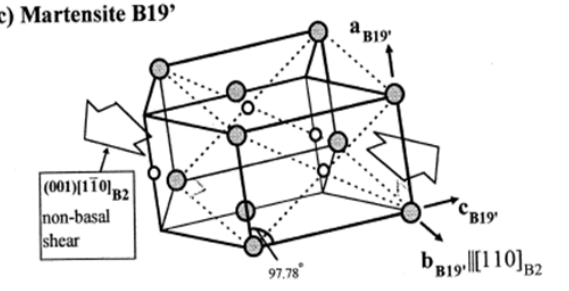

$$
\rightarrow \mathbf{T}_{A}^{\mathrm{B} 2 \rightarrow \mathrm{B} 19 \prime}=\left[\begin{array}{ccc}
0.128 & 0.966 & -1.084 \\
-0.128 & 0.966 & 1.0834 \\
0.951 & 0 . & 0 .
\end{array}\right]
$$




\section{Crystallography of $\mathrm{B} 2 \rightarrow \mathrm{B} 19$ ' transformation - without PTMC - (2/4)}

3. The distortion matrix $\mathbf{F}^{\mathrm{B} 2}$ results from the fundamental equation

$$
\begin{aligned}
& \mathbf{C}^{\mathrm{B} 19 \prime \rightarrow \mathrm{B} 2}=\mathbf{T}^{\mathrm{B} 19 \prime \rightarrow \mathrm{B} 2} \mathbf{F}^{\mathrm{B} 2} \text { where } \mathbf{C}^{\mathrm{B} 19 \prime \rightarrow \mathrm{B} 2}=\left(\mathbf{C}^{\mathrm{B} 2 \rightarrow \mathrm{B} 19 \prime}\right)^{-1} \text { and } \mathbf{T}^{\mathrm{B} 19 \prime \rightarrow \mathrm{B} 2}=\left(\mathbf{T}^{\mathrm{B} 2 \rightarrow \mathrm{B} 19 \prime}\right)^{-1} \\
& \rightarrow \mathbf{F}_{A}^{\mathrm{B} 2}=\left[\begin{array}{ccc}
1.025 & -0.059 & 0.128 \\
-0.059 & 1.025 & -0.128 \\
0 . & 0 . & 0.951
\end{array}\right] . \text { During our st } \\
& \text { martensitic }
\end{aligned}
$$

\begin{tabular}{|c|c|c|c|c|c|}
\hline OR & & Distortior & Matrix F & & Eigenvectors of $\left(F^{B 2}\right)^{*}=\left(F^{B 2}\right)^{-t}$ \\
\hline A & $\mathbf{F}_{A}^{\mathrm{B} 2}=$ & {$\left[\begin{array}{c}1.025 \\
-0.059 \\
0 .\end{array}\right.$} & $\begin{array}{c}-0.059 \\
1.025 \\
0 . \\
\end{array}$ & $\left.\begin{array}{c}0.128 \\
-0.128 \\
0.951\end{array}\right]$ & $\{0,0,1\},\{1,1,0\},\{0.417,-0.417,0.807\}$ \\
\hline $\mathrm{AQ}$ & $\mathbf{F}_{A Q}^{\mathrm{B} 2}=$ & {$\left[\begin{array}{c}1.025 \\
-0.059 \\
-0.0016\end{array}\right.$} & $\begin{array}{c}-0.059 \\
1.025 \\
0.0016 \\
\end{array}$ & $\left.\begin{array}{c}0.130 \\
-0.130 \\
0.951\end{array}\right]$ & $\{0.012,-0.012,0.999\},\{1,1,0\},\{\mathbf{1},-\mathbf{1}, \mathbf{2}\}$ \\
\hline C & $\mathbf{F}_{C}^{\mathrm{B} 2}=$ & $=\left[\begin{array}{c}1.021 \\
-0.055 \\
0.057\end{array}\right.$ & $\begin{array}{c}-0.055 \\
1.021 \\
-0.057 \\
\end{array}$ & $\left.\begin{array}{c}0 . \\
0 . \\
0.958\end{array}\right]$ & $\{-0.397,0.397,0.827\},\{1,1,0\},\{-1,1,0\}$ \\
\hline CQ & $\mathbf{F}_{C Q}^{\mathrm{B} 2}=$ & {$\left[\begin{array}{c}1.022 \\
-0.056 \\
0.083\end{array}\right.$} & $\begin{array}{c}-0.056 \\
1.022 \\
-0.083 \\
\end{array}$ & $\left.\begin{array}{c}0.054 \\
-0.054 \\
0.966\end{array}\right]$ & $\{-1,1,2\},\{1,1,0\},\{0.642,-0.642,0.420\}$ \\
\hline I & $\mathbf{F}_{I}^{\mathrm{B} 2}=$ & {$\left[\begin{array}{c}1.020 \\
-0.118 \\
0.0287\end{array}\right.$} & $\begin{array}{c}0 . \\
1.0268 \\
0 .\end{array}$ & $\left.\begin{array}{c}0.0939 \\
-0.135 \\
0.954\end{array}\right]$ & $\{0.293,0 .,-0.956\},\{0.713,0.125,0.690\},\{1,0,1\}$ \\
\hline
\end{tabular}

4. We calculate $\mathbf{F}^{\mathrm{B} 2}$ for all the ORs we determined in EBSD

During our studies of martensitic transformations, we came to think that the habit plane is not fully invariant but just unrotated by the distortion.

Mathematically, it is a eigenvectors of the reciprocal distortion matrix.

List of expected habit planes 
For OR AQ (the natural OR) the lowest index expected habit planes is $(1 \overline{1} 2)_{B 2} / /(10 \overline{1})_{B 19^{\prime}}$ Is this "prediction" in agreement with the EBSD maps?

Trace of the $(10 \overline{1})_{\mathrm{B} 19^{\prime}}$ planes (plotted by ARPGE)

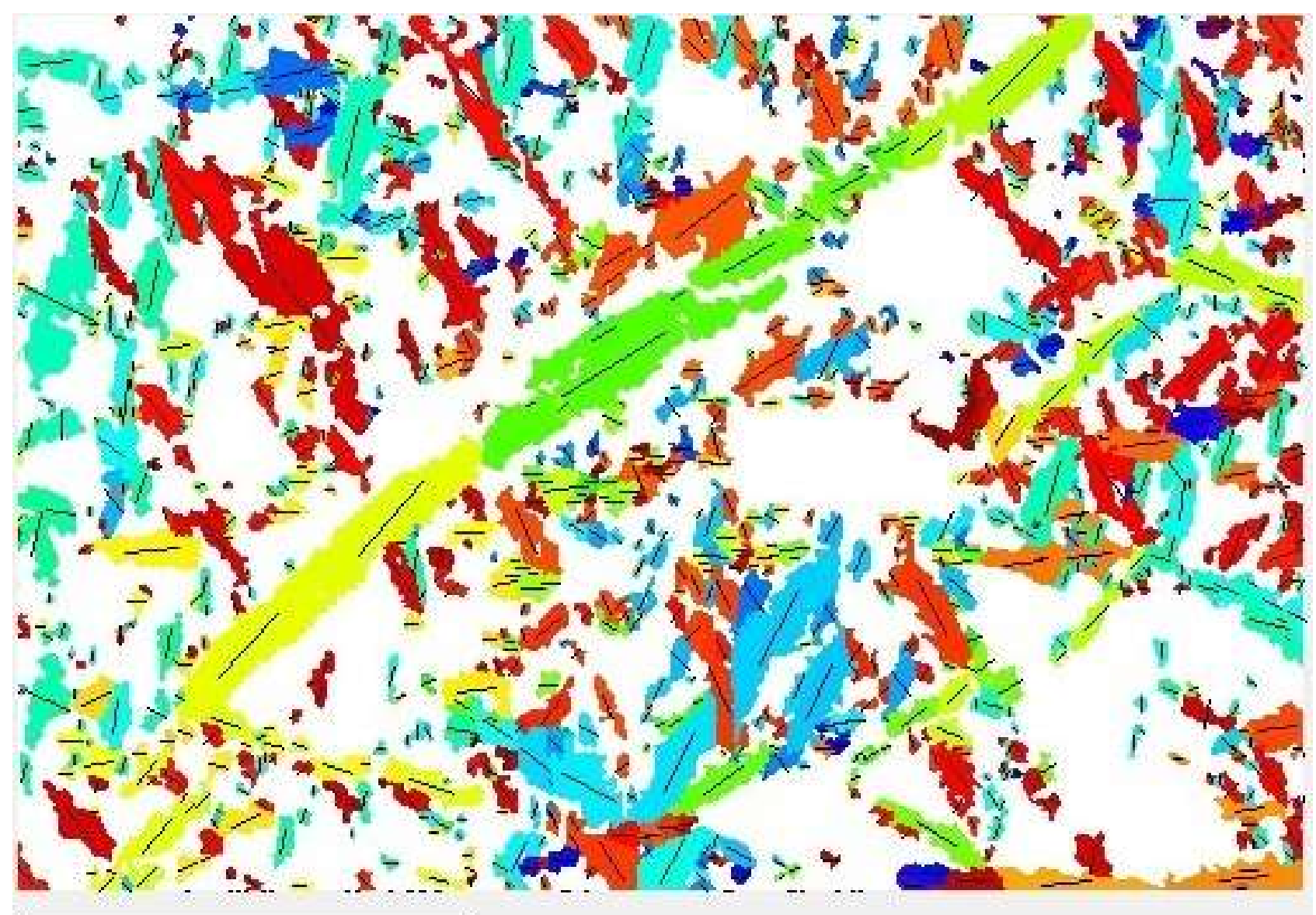

$10 \mu \mathrm{m}$ 
Crystallography of B2 $\rightarrow B 19$ ' transformation - without PTMC - (4/4)

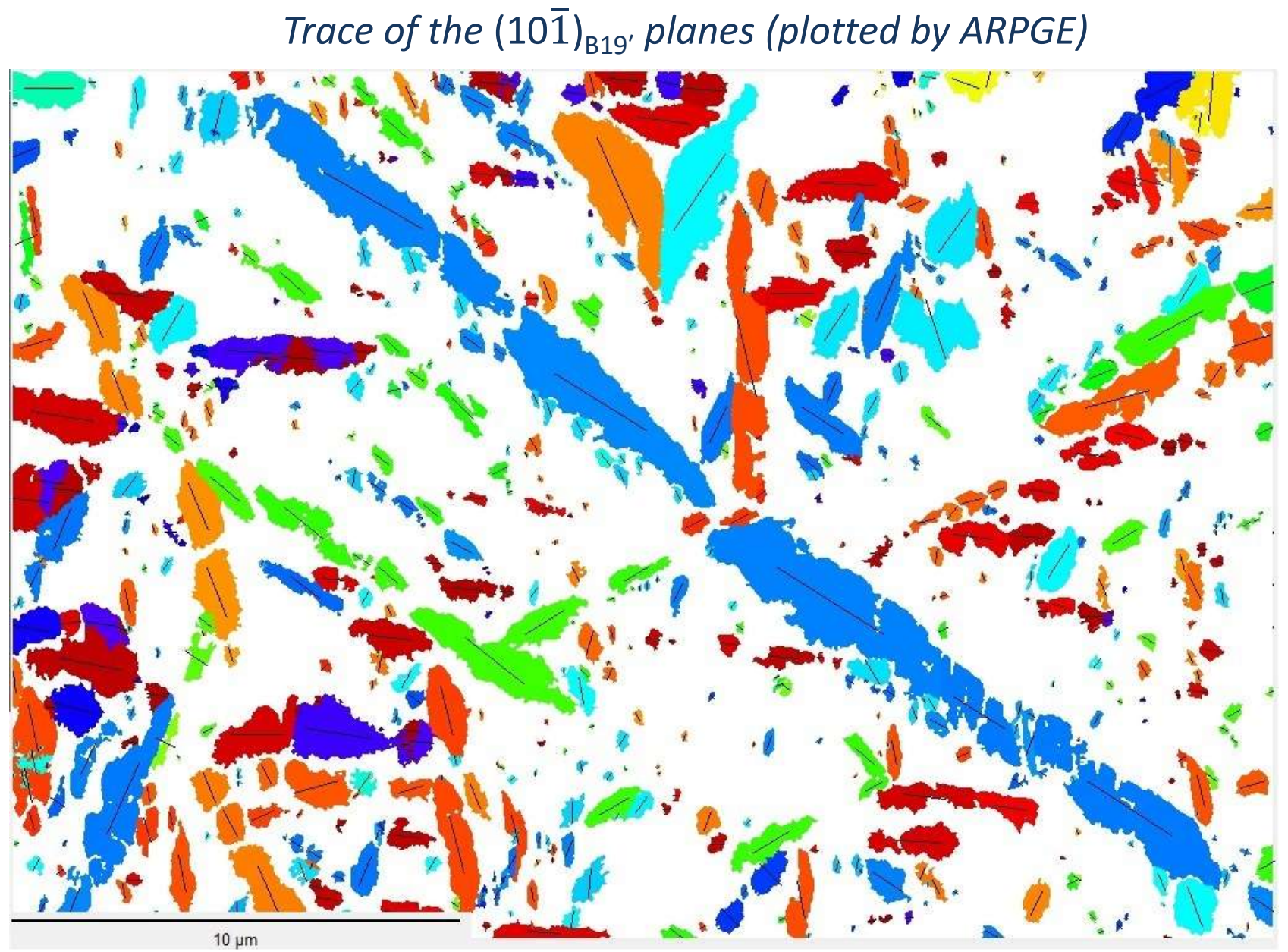

Very good agreement, even better than with the experimental habit planes reported in literature $(0.39,0.48,0.78)_{\mathrm{B} 2}$ or the habit plane $(0.215,0.405,0.888)_{\mathrm{B} 2}$ calculated by PTMC 
For me, the OR AQ is the "natural" OR, and the other ORs are "closing-gap" ORs. Let me explain with a simple 2D square $(\gamma)$-parallelogram $(\alpha)$ transformation

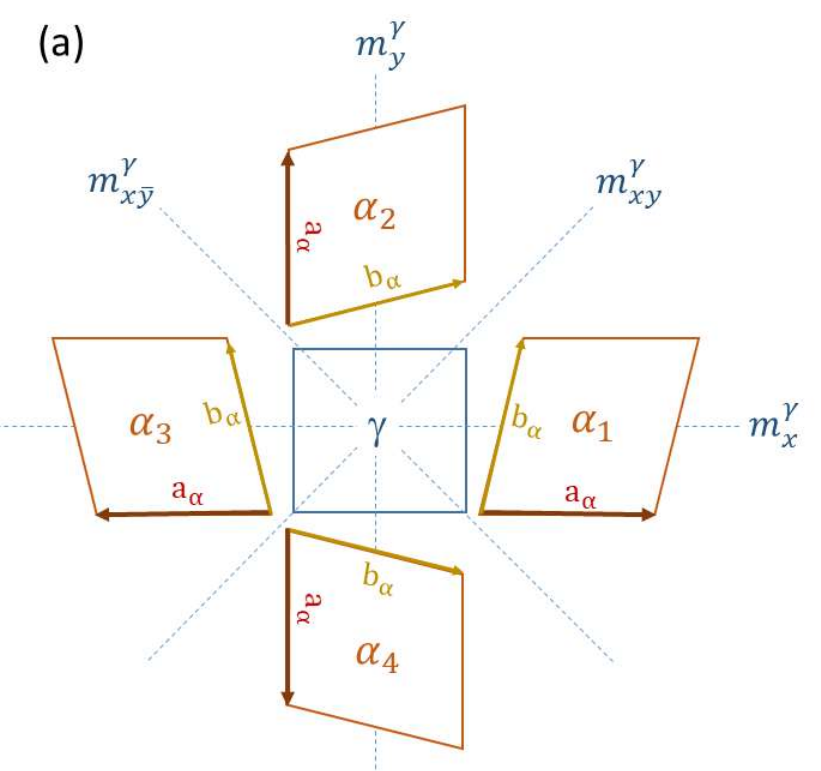

(b)

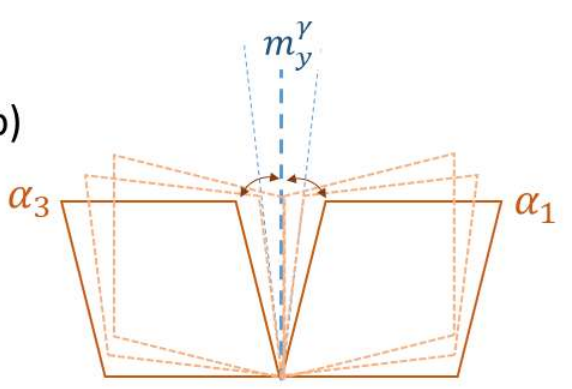

(c)

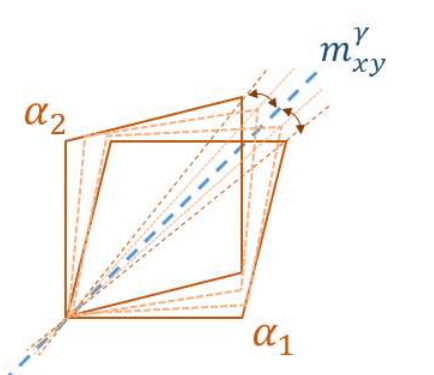

(a) If we assume that $\mathbf{a}_{\alpha} / / \mathbf{a}_{\gamma}$ is the "natural" OR, there are four orientation variants. However, there exist incompatibilities between them. The incompatibilities can be accommodated by closing-gap ORs linked to the natural OR by continuous distortion fields

(b) - Closing-gap OR $(1,0)_{\alpha 1} / /(1,0)_{\alpha 3} / /(1,0)_{\gamma}$ and associated rotation gradients between the variants $\alpha_{1}$ and $\alpha_{3}$. This new OR "cures" the broken symmetry $m_{y}^{\gamma}$.

(c) - Closing-gap OR $(\overline{1}, 1)_{\alpha 1} / /(\overline{1}, 1)_{\alpha 2} / /(\overline{1}, 1)_{\gamma}$ and associated rotation gradients between the variants $\alpha_{1}$ and $\alpha_{2}$. This new OR "cures" the broken symmetry $m_{x y}^{\gamma}$. 
For NiTi alloys, the closing-gap ORs are:

- OR A is the closing-gap OR from AQ that "cures" the lost mirror symmetry on a plane $(001)_{\mathrm{B} 2}$. It restores the contact between two AQ distortion variants that are linked by the common correspondence plane $(001)_{\mathrm{B} 2} \rightarrow(100)_{\mathrm{B} 19^{\prime}}$.

- OR C obeys the same rule and can be understood as the closing-gap OR that re-establishes the contact between the correspondence direction $[001]_{\mathrm{B} 2} \rightarrow[100]_{\mathrm{B} 19^{\prime}}$. The continuum A-C is the elastic accommodation between the natural OR AQ and its two derivatives A and C.

- We also think that the ORs CQ and I derive from other lost symmetry elements such as the $<110\rangle_{\mathrm{B} 2}$ two fold axes

\section{PTMC vs our approach:}

- The PTMC also uses the notion of compatibility between the variants, but the PTMC has never considered a natural OR. Actually, ORs are just adjustment parameters in PTMC.

- The PTMC imposes that a simple shear link (rank-one connection) between the variants.

- Our approach is global (PTMC is local).

- It allows us to understand how the global integrity of the structure of variants is maintained thanks to the natural ORs and its complementary "closing-gap" ORs.
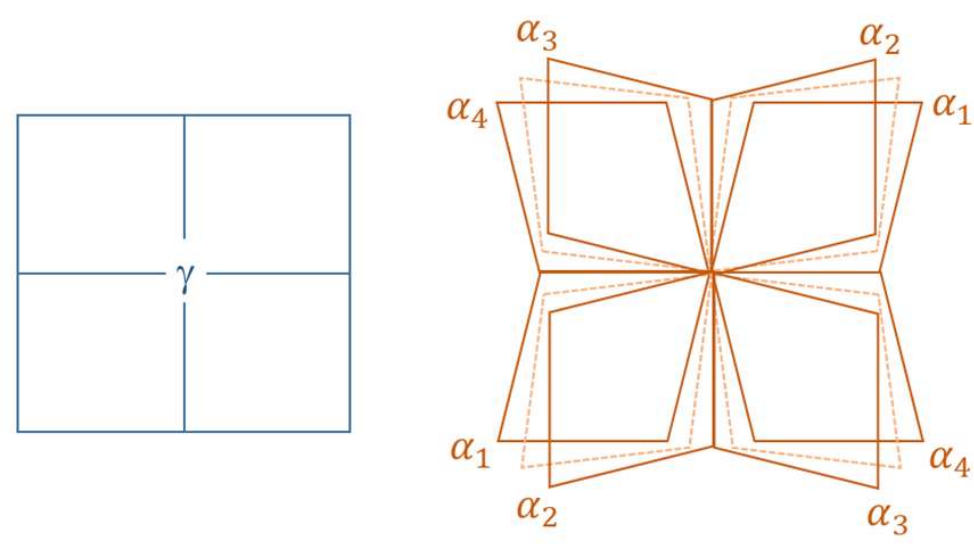


\section{Conclusion}

\section{Experimental results:}

- The complex and intricate microstructure of B19' martensite in shape memory NiTi alloys is generally studied by TEM and explained with the PTMC.

- Here, we used EBSD and TKD to investigate a polycrystalline NiTi alloy composed of B19' martensite.

- The EBSD maps show the martensite plates and reveal the coexistence of different ORs.

- The TKD maps show the "twins" and confirm the continuum of orientations suspected from EBSD.

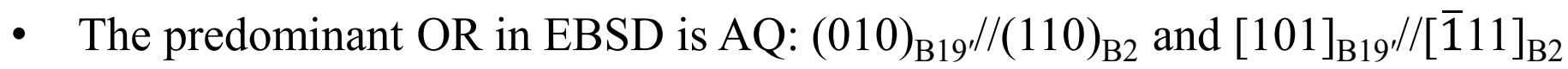

- Other co-existent ORs were observed (A, C, CQ, I).

\section{Interpretation:}

- The experimental results are interpreted with an approach alternative to PTMC.

- The OR AQ is the "natural" OR since the dense directions and dense planes of B2 and B19' phases are parallel.

- The ORA/AQ was used to automatically reconstruct the prior parent B2 grains in the EBSD and TKD maps.

- From the distortion matrix associated with the OR AQ, we calculated that the habit plane could be $(1 \overline{1} 2)_{\mathrm{B} 2} / /(10 \overline{1})_{\mathrm{B} 19^{\prime}}$

- The traces of these planes are in good agreement with the EBSD maps.

- The ORs A, C, CQ and I are "closing-gap" ORs derived from the natural OR to allow the compatibility between the distortion variants.

- Each of them restores a parent symmetry element between the variants that was lost by distortion but preserved by correspondence. 
For more details:

\author{
Open Access Aricie \\ What EBSD and TKD Tell Us about the Crystallography \\ of the Martensitic B2-B19' Transformation in NiTi Shape \\ Memory Alloys \\ by $\mathbb{Q}$ Cyril Cayron $\square$ \\ Laboratory of ThermoMechanical Metallurgy (LMTM), PX Group Chair, Ecole Polytechnique Fédérale de Lausanne (EPFL), rue \\ de la Maladière 71b, 2000 Neuchâtel, Switzerland \\ Crystals 2020, 10(7), 562; https://doi.org/10.3390/cryst10070562
}

\title{
Acknowledgments:
}

This work was helped by the arrival of our new SEM equipped with EBSD. This equipment was financed at $50 \%$ by the SNSF R'Equip project (n 206021 182981) and 50\% by EPFL. I also would like to thank Prof. Roland Logé, director of LMTM, and PX Group who chairs the laboratory. 